\title{
Características edáficas y topográficas asociadas con el crecimiento en volumen de Gmelina arborea Roxb, en Tlatlaya, Estado de México
}

\author{
Edaphic and topographic characteristics associated with growth in \\ volume of Gmelina arborea Roxb, in Tlatlaya, Mexico State
}

\section{Ricardo Telles Antonio', Eduardo Alanís Rodríguez²*, Javier Jiménez Pérez², Oscar Alberto Aguirre Calderón², Eduardo Javier Treviño Garza² y Héctor Manuel de los Santos Posadas ${ }^{3}$}

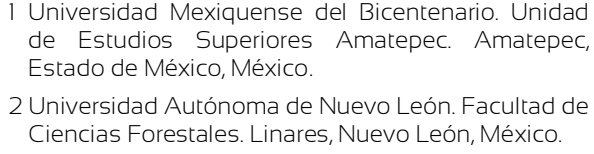

2 Universidad Autónoma de Nuevo León. Facultad de

Ciencias Forestales. Linares, Nuevo León, México.

\section{RESUMEN}

Gmelina arborea es una especie de rápido crecimiento y fácil adaptación a diversidad de condiciones de sitio; el objetivo del estudio fue analizar las características edáficas y topográficas asociadas con el crecimiento en volumen fustal de G. arborea establecida en el Estado de México y desarrollar un modelo para estimar el volumen fustal. La investigación fue desarrollada en ocho parcelas permanentes de muestreo de forma circular $\left(400 \mathrm{~m}^{2}\right)$, donde se extrajeron 5 sub-muestras de suelo de cada parcela, las cuales fueron mezcladas para formar una muestra final de 500 gramos. El análisis de las características edáficas se hizo con base en la NOM-021-RECNAT-2000 y datos de altitud, pendiente y profundidad. Para el análisis de varianza y prueba de medias, se efectúo un análisis de componentes principales y posteriormente, una regresión lineal múltiple; a través del coeficiente de correlación, se compararon las correlaciones existentes entre las variables elegidas con el paquete estadístico $\mathrm{R} \circledast$. El modelo resultante tomó como variables predictoras la conductividad hidráulica, los nitratos y la relación $\mathrm{Ca} / \mathrm{Mg}$; este modelo explicó $71.6 \%$ de la variabilidad observada en el volumen fustal $\left(\mathrm{R}^{2}{ }_{\text {ajustado }}=0.716\right)$. De acuerdo con el resultado de varianza, estas variables no mostraron diferencias significativas. Se concluye que la variación de las características edáficas y topográficas influye en el crecimiento del volumen fustal. Es indispensable conocer la autoecología de la especie, la cual está asociada a condiciones climáticas y edafológicas, así como a la distribución altitudinal.

PALABRAS CLAVE: Gmelina arborea, nutrimentos, plantación forestal, productividad, suelos.

\section{ABSTRACT}

Gmelina arborea is a fast-growing species easily adaptable to a diversity of site conditions; the objective of the study was to analyze the edaphic and topographic characteristics associated with the growth of $G$. arborea established in Mexico State and to develop a model to estimate the stem volume. The research was carried out in eight permanent sampling plots of circular shape $\left(400 \mathrm{~m}^{2}\right)$, where 5 soil subsamples were extracted from each plot, which were mixed to form a final sample of 500 grams. The analysis of edaphic characteristics was made based on NOM-021-RECNAT-2000 and data on altitude, slope, and depth. For the analysis of variance and test of means, a principal component analysis was carried out, followed by a multiple linear regression; the correlation coefficient was used to compare the correlations between the variables chosen with the $\mathrm{R} \AA$ statistical package. The resulting model took hydraulic conductivity, nitratesand the $\mathrm{Ca} / \mathrm{Mg}$ ratio as predictor variables; this model explained $71.6 \%$ of the variability observed in the volume of the trunk $\left(\mathrm{R}^{2}\right.$ adjusted $\left.=0.716\right)$. According to the variance results, these variables did not show significant differences. It is concluded that the variation of the edaphic and topographic characteristics influences the growth of the volume of the canopy. It is essential to know the autoecology of the species, which is associated with climatic and edaphological conditions, as well as altitudinal distribution.

KEYWORDS: Gmelina arborea, nutrients, forest plantation, productivity, soils. 


\section{INTRODUCCIÓN}

Gmelina arborea Roxb. es una especie de rápido crecimiento a lo largo del turno y de fácil adaptación a una diversidad de condiciones de sitio; comúnmente es utilizada para el establecimiento de plantaciones comerciales (Rojas y Murillo, 2004; Indira, 2006; Moya-Roque et al., 2010). Debido a su alta productividad maderable, G. arborea ofrece a los productores e inversionistas un rápido retorno del capital (Wee, Li, Dvorak, y Hong, 2012). Por las características tecnológicas que presenta su madera, es de fácil trabajabilidad (Gonzáles y Serrano, 2004) y buen acabado; igualmente, puede ser utilizada en muebles de alta calidad (Rejón y Romero, 2004), construcción, tableros contrachapados, lápices, etc. De igual forma, posee un alto potencial para la elaboración de otros productos como tableros de fibras, tableros de partículas y pulpa para papel (Gonzáles y Serrano, 2004).

México cuenta con 270000 ha de plantaciones forestales comerciales; de las principales especies maderables establecidas, G. arborea cuenta con 24061 ha (Comisión Nacional Forestal [Conafor], 2014). En el Estado de México existe una tendencia de adición de 250 ha de $G$. arborea establecidas por año, con una superficie total de 1248 ha al 2018 (Protectora de Bosques del Estado de México [Probosque], 2019).

En Colombia, Barrios et al. (2011) caracterizaron químicamente el suelo de una plantación de G. arborea de cuatro años de edad, establecieron dosis de fertilización y concluyeron que el máximo incremento promedio anual en volumen total sin corteza alcanza 15\% en relación con las parcelas testigo.

El pH del suelo se ha correlacionado positivamente con la altura total y con el diámetro normal al determinar la estructura de un bosque tropical en predominancia de Tectona grandis L. f. en la India (Kumar, Patel, Kumar y Kumar, 2011). Bueis (2012) desarrolló un modelo discriminante para predecir la productividad de Pinus sylvestris L. con parámetros topográficos, edáficos (físicos, químicos y bioquímicos) y climáticos. En plantaciones de
T. grandis establecidas en Nayarit, Salcedo-Pérez et al. (2014) determinaron que un suelo con poca materia orgánica (MO) influye en la capacidad de intercambio catiónico (CIC), que deriva en un menor desarrollo tanto en altura como en diámetro normal y que el contenido de potasio $(\mathrm{K})$ y fósforo $(\mathrm{P})$ inciden de manera positiva en el crecimiento de la especie.

Salcedo-Pérez et al. (2019) establecieron que suelos con $\mathrm{pH}$ de entre 6 y $7, \mathrm{CIC}>30 \mathrm{cmol}^{(+)} \mathrm{kg}$ y materia orgánica $>2.5 \%$ se asocian a las plantaciones de mejor productividad de $T$. grandis y concluyeron que, en México, el éxito en la productividad de plantaciones de especies exóticas depende, esencialmente de las propiedades del suelo. Por su parte Ruiz-Blandon et al. (2020) estipularon que los mejores parámetros dasométricos y producción de biomasa de $G$. arborea, se asociaron a suelos con $\mathrm{pH}>6$ y relación $\mathrm{C} / \mathrm{N}>20$, y que el óptimo rendimiento dependerá del arreglo forestal, el manejo y las características edáficas.

\section{OBJETIVOS}

Los objetivos del estudio fueron analizar las características físicas y químicas del suelo, así como las topográficas asociadas con el crecimiento en volumen fustal de una plantación de Gmelina arborea establecida en Tlatlaya, Estado de México, además de desarrollar un modelo para estimar el volumen fustal.

\section{MATERIALES Y MÉTODOS}

\section{Área de estudio}

El estudio se desarrolló en una plantación forestal comercial de G. arborea ubicada en el predio "Las Piñas", municipio de Tlatlaya, Estado de México (Fig. 1). El clima es (Aw1) cálido subhúmedo con lluvias en verano (García, 2004), temperatura media anual de $18{ }^{\circ} \mathrm{C}$ a $28{ }^{\circ} \mathrm{C}$ y precipitación anual que fluctúa entre los $1000 \mathrm{~mm}$ y los $1500 \mathrm{~mm}$ (Instituto Nacional de Estadística Geografía e Informática [Inegi], 2009). Los suelos predominantes son del grupo Regosol (Inegi, 2009). 


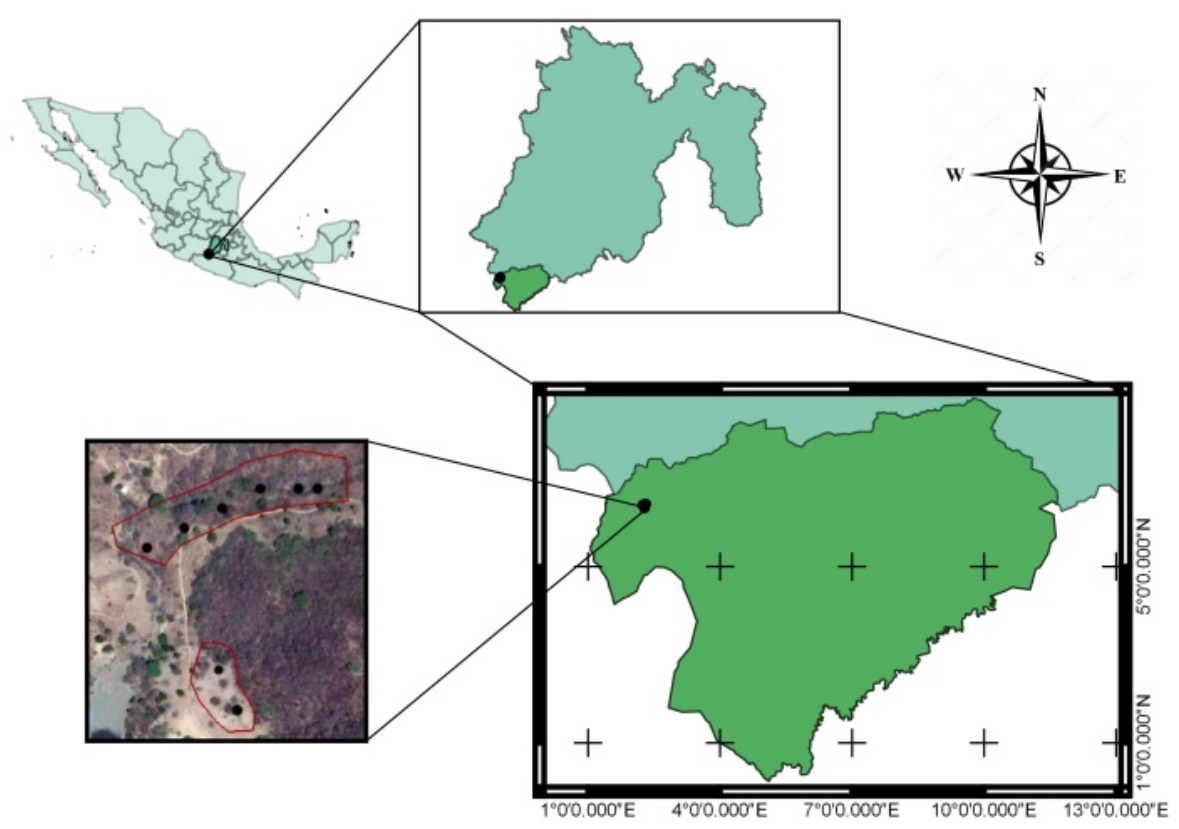

FIGURA 1. Ubicación de la PFC de G. arborea establecida en Tlatlaya, Estado de México.

La toma de datos de campo se realizó en el año 2017 a los tres años de edad de la plantación, la superficie de la plantación fue de ocho hectáreas. Los árboles estaban plantados bajo un arreglo tipológico en tresbolillo con un espaciamiento de $3.10 \mathrm{~m} \times 3.10 \mathrm{~m}$ y con una densidad correspondiente a 1040 árboles por hectárea. Se establecieron ocho parcelas permanentes de muestreo de forma circular $\left(400 \mathrm{~m}^{2}\right)$ y a los árboles se les registraron datos de diámetros a $0.30 \mathrm{~m}$ sobre el nivel del suelo, diámetro normal $(1.30 \mathrm{~m})$ y altura total. Estas mediciones se hicieron con una forcípula Haglof Sweden ${ }^{\circledR}$ y con un hipsómetro Nikon Forestry Proß. Adicionalmente, se caracterizó el estado fitosanitario y el grado de supervivencia de los árboles. Con los datos obtenidos de este inventario, se generó información de área basal y de volumen fustal con la ecuación de cubicación de Rodríguez y Castañeda (2014) (Ec. 1).

$$
V=\frac{\pi}{4} \cdot D^{2} \cdot h \cdot f f
$$

Donde:

$V=$ Volumen $\left(\mathrm{m}^{3}\right)$

$D=$ Diámetro normal $(\mathrm{m})$

$h=$ Altura total $(\mathrm{m})$

$f f=$ Factor de forma $(0.46)$

\section{Datos edáficos y topográficos}

Para determinar las características físico-químicas del suelo, se extrajeron cinco sub-muestras de cada parcela, se distribuyeron con base en un muestreo diagonal de acuerdo con los puntos cardinales $\mathrm{N}, \mathrm{S}, \mathrm{E}$ y W y una al centro de la parcela hasta una profundidad de $30 \mathrm{~cm}$ (Barrios et al., 2011; Salcedo-Pérez et al., 2019). Con las cinco sub-muestras se procedió a formar una muestra compuesta que fue mezclada homogéneamente y se dividió en cuatro partes para obtener una muestra final de $500 \mathrm{~g}$, misma que fue rotulada con datos de identificación (Salcedo-Pérez et al., 2014).

Las características físicas analizadas de cada muestra fueron: clase textural, punto de saturación, capacidad de 
campo, punto de marchitez permanente, conductividad hidráulica, y densidad aparente, con base en los métodos descritos por la NOM-021-RECNAT-2000 (Secretaría del Medio Ambiente y Recursos Naturales [Semarnat], 2002; Salcedo-Pérez et al., 2019).

Las características químicas analizadas de cada muestra fueron: $\mathrm{MO}, \mathrm{P}, \mathrm{K}, \mathrm{Ca}, \mathrm{Mg}, \mathrm{Na}$, hierro (Fe), Zinc ( $\mathrm{Zn}$ ), manganeso $(\mathrm{Mn})$, cobre $(\mathrm{Cu})$, boro $(\mathrm{B})$, aluminio $(\mathrm{Al})$, azufre (S), nitratos (N-NO3), $\mathrm{pH}$, carbonatos totales, salinidad y relación de cationes: $\mathrm{Ca} / \mathrm{K}, \mathrm{Mg} / \mathrm{K}, \mathrm{Ca}+\mathrm{Mg} / \mathrm{K}$, $\mathrm{Ca} / \mathrm{Mg}$, CIC, Ca, $\mathrm{Mg}, \mathrm{K}$ y Na, con base en los métodos descritos por la NOM-021-RECNAT-2000 (SEMARNAT, 2002); conjuntamente se obtuvieron datos topográficos de altitud $(\mathrm{m})$, pendiente $\left({ }^{\circ}\right)$ y profundidad $(\mathrm{cm})$ (Ávila, Murillo, Murillo y Sandoval, 2015).

\section{Análisis estadístico}

Se realizó un análisis de varianza (procedimiento ANOVA) de un factor $y$, posteriormente, a las variables que presentaron diferencia significativa, se les aplicó la prueba de medias de Tukey $(\mathrm{p}<0.05)$ como comparador múltiple, para verificar posibles diferencias significativas entre las variables (Rubio y Berlanga, 2012): dasométricas, físicoquímicas y topográficas (Salcedo-Pérez et al., 2014; González-Rojas, Murillo-Cruz y Ávila-Arias, 2016; SalcedoPérez et al., 2019).

La información se sometió a un análisis de componentes principales (ACP) como técnica exploratoria sobre las variables agrupadas por su tipo (edáficas físicas, edáficas químicas y topográficas) para reducir la dimensionalidad de los datos y con el propósito de seleccionar aquellas no correlacionadas entre sí que recogieran la mayor variabilidad de los datos (Thiers y Gerding, 2007).

El ACP se efectuó por grupos de variables ya que se considera que las correlaciones existirán mayoritariamente entre variables del mismo tipo. Ante ello, se tomó como criterio combinado de elección del número de componentes principales a considerar en cada análisis que el conjunto de estas recogiera al menos $45 \%$ de la varianza.
La relevancia de las variables en cada componente principal se evaluó a través de su valor de contribución (autovalor), el cual es más significativo en la medida que se aproxima al valor |1 | (Isebrands y Crow, 1975; Guisande, Vaamonde y Barreiro, 2013).

De cada uno de los componentes, se identificó el orden en que las diferentes variables de las parcelas están asociadas con el crecimiento en volumen fustal de $G$. arborea (Thiers y Gerding, 2007).

Se comprobó la normalidad de las variables seleccionadas a través del ACP mediante la prueba ShapiroWilk. Las variables que no presentaban una distribución normal se excluyeron del análisis de regresión lineal múltiple (Mariño-Macana y Rodríguez-Melo, 2010). Se utilizó el coeficiente de correlación de Pearson para analizar las similitudes existentes entre las variables topográficas y edáficas seleccionadas entre sí (Adeboye et al., 2011; Kumar et al., 2011; Salcedo-Pérez et al., 2014). Todos los análisis estadísticos fueron realizados con el paquete estadístico $R \mathbb{}$ versión 3.14 (Santana y Farfán, 2014).

Para verificar el cumplimiento de los supuestos de la regresión, se determinaron la normalidad de los residuos (Shapiro-Wilk), la homogeneidad de varianzas de los residuos (Breusch-Pagan) y la independencia de los residuos (Durbin-Watson).

Se analizó la distribución de los residuales mediante método gráfico de la ecuación de regresión de volumen fustal de G. arborea, para observar la distribución de los residuos, tal como lo recomiendan Álvarez et al. (2003).

\section{Resultados}

Las características edáficas físicas de las parcelas de la plantación de $G$. arborea mostraron que la clase textural es francosa $(\mathrm{C})$, franco arcillosa $(\mathrm{Cr})$ y franco arcillo arenosa (Cra), la cual clasifica a los suelos de las parcelas como francosos (NOM-021-RECNAT-2000) (Tabla 1). Las parcelas presentan entre $28 \%$ y $40.6 \%$ de arcilla a excepción de la parcela 6 que contiene $51.2 \%$. Las características edáficas químicas analizadas se resumen en la tabla 2 , las topográficas en la tabla 3 y las dasométricas en la tabla 4. 
TABLA 1. Comparación de medias por Tukey de las características edáficas físicas analizadas en las parcelas de G. arborea establecida en Tlatlaya, Estado de México a los tres años de edad.

\begin{tabular}{cccc}
\hline Característica física & ${ }^{*}$ Medias & Pr>F & Clasificación NOM-O21-RECNAT-2OOO \\
\hline PS (\%) & $37.13 \pm 7.25 \mathrm{ab}$ & 0.000 & -- \\
CC (\%) & $19.10 \pm 3.93 \mathrm{ab}$ & 0.000 & Media baja 12-20 \\
PMP (\%) & $11.72 \pm 2.33 \mathrm{ab}$ & 0.000 & -- \\
$\mathrm{CH}\left(\mathrm{cm} \mathrm{h}^{-1}\right)$ & $6.01 \pm 1.88$ & 0.198 & Moderadamente Alto \\
DA $\left(\mathrm{g} \mathrm{cm}^{-3}\right)$ & $1.26 \pm 0.10 \mathrm{ab}$ & 0.002 & Francosos 1.20-1.32 \\
\hline
\end{tabular}

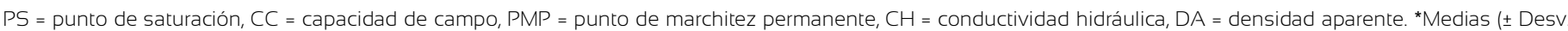
estándar).

Letras distintas en hileras indican diferencias significativas entre las parcelas, determinadas mediante la prueba de Tukey ( $<<0.05)$.

TABLA 2. Comparación de medias por Tukey de las características edáficas químicas analizadas en las parcelas de G. arborea establecida en Tlatlaya, Estado de México a los tres años de edad.

\begin{tabular}{|c|c|c|c|c|c|}
\hline \multicolumn{2}{|c|}{ Característica Química } & $\begin{array}{l}\text { Unidad de } \\
\text { medida }\end{array}$ & **Medias & $\operatorname{Pr}>\mathrm{F}$ & $\begin{array}{c}\text { Clasificación } \\
\text { NOM-O21-RECNAT-2000 }\end{array}$ \\
\hline \multicolumn{2}{|l|}{${ }^{*} \mathrm{MO}$} & $\%$ & $2.67 \pm 0.45$ & 0.057 & Medio 0.6-3.5 \\
\hline \multicolumn{2}{|l|}{$P$} & & $6.94 \pm 6.43$ & 0.360 & Bajo $<15$ \\
\hline \multicolumn{2}{|l|}{ K } & & $118.2 \pm 58.72 \mathrm{ab}$ & 0.000 & Bajo 100-150 \\
\hline \multicolumn{2}{|l|}{$\mathrm{Ca}$} & & $2155.63 \pm 1230.55 a b$ & 0.000 & Alto $>2000$ \\
\hline \multicolumn{2}{|l|}{$\mathrm{Mg}$} & & $393.75 \pm 271.81 \mathrm{ab}$ & 0.002 & Alto $>180$ \\
\hline \multicolumn{2}{|l|}{$\mathrm{Na}$} & & $15.47 \pm 5.20 a b$ & 0.000 & -- \\
\hline \multicolumn{2}{|l|}{$\mathrm{Fe}$} & ח & $26.85 \pm 8.50 \mathrm{ab}$ & 0.000 & Muy alto $>2.10$ \\
\hline \multicolumn{2}{|l|}{$\mathrm{Zn}$} & ppim & $1.23 \pm 0.77 a b$ & 0.003 & Medio 0.80-1.29 \\
\hline \multicolumn{2}{|l|}{$\mathrm{Mn}$} & & $32.09 \pm 8.80 \mathrm{ab}$ & 0.000 & Muy Alto >2.10 \\
\hline \multicolumn{2}{|l|}{$\mathrm{Cu}$} & & $4.24 \pm 1.17$ & 0.796 & Muy Alto >2.10 \\
\hline \multicolumn{2}{|l|}{ B } & & $0.27 \pm 0.05 a b$ & 0.000 & Muy bajo $<0.39$ \\
\hline \multicolumn{2}{|l|}{ S } & & $2.19 \pm 1.03 \mathrm{ab}$ & 0.029 & Medio 2-10 \\
\hline \multicolumn{2}{|l|}{$\mathrm{N}-\mathrm{NO} 3$} & & $4.49 \pm 1.09$ & 0.995 & Muy bajo 0-10 \\
\hline \multicolumn{2}{|l|}{$\mathrm{pH}$} & -- & $6.50 \pm 0.71 \mathrm{ab}$ & 0.045 & Moderadamente ácido 5.1-6.5 \\
\hline \multicolumn{2}{|c|}{ Carbonatos Totales } & $\%$ & $0.62 \pm 1.05 \mathrm{ab}$ & 0.001 & -- \\
\hline \multicolumn{2}{|l|}{ Salinidad (CE) } & $\mathrm{dS} \mathrm{m}^{-1}$ & $0.15 \pm 0.04 \mathrm{ab}$ & 0.000 & No salino 0-2.0 \\
\hline \multirow{4}{*}{$\begin{array}{l}\text { Relación entre } \\
\text { cationes }\end{array}$} & $\mathrm{Ca} / \mathrm{K}$ & \multirow{9}{*}{ meq/100g } & $40.42 \pm 22.32 \mathrm{ab}$ & 0.001 & - \\
\hline & $\mathrm{Mg} / \mathrm{K}$ & & $12.75 \pm 8.26 \mathrm{ab}$ & 0.023 & -- \\
\hline & $\begin{array}{l}\mathrm{Ca}+\mathrm{Mg} / \\
\mathrm{K}\end{array}$ & & $53.15 \pm 27.46 \mathrm{ab}$ & 0.000 & -- \\
\hline & $\mathrm{Ca} / \mathrm{Mg}$ & & $3.86 \pm 1.48$ & 0.543 & -- \\
\hline \multirow{5}{*}{$\begin{array}{l}\text { Cationes } \\
\text { intercambiables }\end{array}$} & $\mathrm{Ca}$ & & $12.56 \pm 6.98 \mathrm{ab}$ & 0.012 & Alta $>10$ \\
\hline & $\mathrm{Mg}$ & & $3.48 \pm 2.28$ & 0.418 & Alta $>3.0$ \\
\hline & K & & $0.32 \pm 0.14 \mathrm{ab}$ & 0.000 & Media 0.3-0.6 \\
\hline & $\mathrm{Na}$ & & $0.07 \pm 0.03 \mathrm{ab}$ & 0.000 & -- \\
\hline & $\mathrm{CIC}$ & & $16.65 \pm 8.39 a b$ & 0.002 & Media 15-25 \\
\hline
\end{tabular}

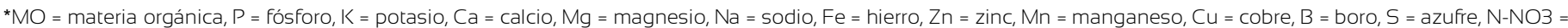
nitratos, $\mathrm{pH}=$ potencial de hidrógeno.

**Medias ( \pm Desv. estándar). Letras distintas en hileras indican diferencias significativas entre las parcelas, determinadas mediante la prueba de Tukey ( $<<0.05)$. 
TABLA 3. Comparación de medias por Tukey de las características topográficas analizadas en las parcelas de G. arborea establecida en Tlatlaya, Estado de México a los tres años de edad.

\begin{tabular}{ccc}
\hline Características topográficas & ${ }^{*}$ Medias & Pr $>\mathrm{F}$ \\
\hline Altitud $(\mathrm{m}$ s.n.m.) & $694.63 \pm 8.38 \mathrm{ab}$ & 0.000 \\
Pendiente $\left({ }^{\circ}\right)$ & $21.39 \pm 7.36 \mathrm{ab}$ & 0.000 \\
Profundidad $(\mathrm{cm})$ & $31.38 \pm 1.86 \mathrm{ab}$ & 0.000 \\
\hline
\end{tabular}

*Medias ( \pm Desv. estándar).

Letras distintas en hileras indican diferencias significativas entre las parcelas, determinadas mediante la prueba de Tukey ( $p<0.05)$.

TABLA 4. Comparación de medias por Tukey de las variables dasométricas de G. arborea establecida en Tlatlaya, Estado de México a los tres años de edad.

\begin{tabular}{ccccc}
\hline Parcela & Diámetro tocón $(\mathrm{cm})$ & Diámetro normal $(\mathrm{cm})$ & Altura total $(\mathrm{m})$ & Volumen fustal $\left(\mathrm{m}^{3} \mathrm{ha}^{-1}\right)$ \\
\hline 1 & $12.01 \pm 2.57 \mathrm{bcd}$ & $9.00 \pm 1.95 \mathrm{abc}$ & $6.72 \pm 0.96 \mathrm{~cd}$ & $0.021 \pm 0.01 \mathrm{abc}$ \\
2 & $11.33 \pm 3.12 \mathrm{abc}$ & $8.25 \pm 2.75 \mathrm{a}$ & $6.04 \pm 1.32 \mathrm{abc}$ & $0.019 \pm 0.02 \mathrm{ab}$ \\
3 & $11.38 \pm 2.05 \mathrm{abc}$ & $8.42 \pm 1.81 \mathrm{ab}$ & $6.37 \pm 0.77 \mathrm{acd}$ & $0.018 \pm 0.01 \mathrm{ab}$ \\
4 & $10.71 \pm 2.11 \mathrm{ab}$ & $7.74 \pm 1.51 \mathrm{a}$ & $5.88 \pm 0.93 \mathrm{ab}$ & $0.014 \pm 0.01 \mathrm{a}$ \\
5 & $11.04 \pm 1.36 \mathrm{abc}$ & $8.34 \pm 1.20 \mathrm{ab}$ & $5.69 \pm 0.50 \mathrm{ab}$ & $0.015 \pm 0.00 \mathrm{ab}$ \\
6 & $13.57 \pm 2.96 \mathrm{~d}$ & $10.23 \pm 2.19 \mathrm{c}$ & $7.11 \pm 1.03 \mathrm{~d}$ & $0.030 \pm 0.02 \mathrm{c}$ \\
7 & $13.27 \pm 1.97 \mathrm{~cd}$ & $9.99 \pm 1.77 \mathrm{bc}$ & $6.36 \pm 0.89 \mathrm{acd}$ & $0.024 \pm 0.01 \mathrm{bc}$ \\
8 & $9.75 \pm 1.80 \mathrm{~b}$ & $7.29 \pm 1.53 \mathrm{a}$ & $5.25 \pm 0.91 \mathrm{~b}$ & $0.011 \pm 0.01 \mathrm{a}$ \\
\hline
\end{tabular}

Medias ( \pm Desv. estándar).

Letras distintas en columnas indican diferencias significativas entre las parcelas, determinadas mediante la prueba de Tukey ( $P<0.05$ )

\section{Análisis de componentes principales}

$\mathrm{El}$ análisis de componentes principales para el conjunto de variables edáficas físicas estudiadas evidenció tres componentes principales, los cuales se presentan en la tabla 5. Se seleccionaron las variables CC, DA y $\mathrm{CH}$ por ser representativas de los componentes principales (Tabla 6).

Con base en los grupos de componentes principales conformados por los tres tipos de variables: edáficas físicas, edáficas químicas y topográficas, se optó por limitar el número de componentes principales a dos por tipo de variable, como lo sugieren Guisande et al. (2013) con la intención de reducir la dimensionalidad de los datos. También para los tres tipos de variables, se generó el círculo de correlación para los dos componentes principales que obtuvieron un valor de contribución (autovalor) aproximado al valor 1 .

En la figura 2 se observa que el CP1 proporcionó mayor peso a las variables edáficas físicas CC, PMP, PS y $\mathrm{CH}$, en orden de importancia. Además, se infiere que están relacionadas con las parcelas 3, 4, 6 y 7 por presentar resultados mínimos y máximos del análisis en laboratorio. El CP2 proporcionó mayor peso a la variable edáfica física DA y está relacionada con las parcelas 5 y 8 que presentaron resultados de DA máximos.

Para el conjunto de variables edáficas químicas estudiadas, se conformaron seis componentes principales (Tabla 7). Se seleccionaron las variables químicas: relación $\mathrm{Ca}+\mathrm{Mg} / \mathrm{K}, \mathrm{Mn}, \mathrm{Ca} / \mathrm{Mg}, \mathrm{N}-\mathrm{N} 03, \mathrm{~S}$ y $\mathrm{Cu}$ por ser representativas de los componentes principales (Tabla 8). 
TABLA 5. Autovalor, porcentaje de varianza y porcentaje de varianza acumulado asociado del análisis de componentes principales de las variables edáficas físicas analizadas en la plantación de G. arborea en Tlatlaya, Estado de México.

\begin{tabular}{cccc}
\hline Componente principal & Autovalor & Porcentaje de varianza & Porcentaje de varianza acumulado \\
\hline 1 & 1.9976 & 79.81 & 79.81 \\
2 & 0.9989 & 19.96 & 99.77 \\
3 & 0.1074 & 0.23 & 99.99 \\
\hline
\end{tabular}

TABLA 6. Coeficientes seleccionados y los primeros tres componentes principales del ACP de las variables edáficas físicas de la plantación de G. arborea en Tlatlaya, Estado de México.

\begin{tabular}{cccc}
\hline Variables & CP1 & CP2 & CP3 \\
\hline *PS (\%) & -0.5003 & 0.0014 & -0.2838 \\
CC (\%) & -0.5004 & 0.0021 & -0.2808 \\
PMP (\%) & -0.5003 & 0.0080 & -0.2995 \\
CH (cm h$)$ & 0.4962 & -0.0934 & -0.8631 \\
DA $\left(\mathrm{g} \mathrm{cm}^{-3}\right)$ & 0.0523 & 0.9956 & -0.0775 \\
\hline
\end{tabular}

*PS = punto de saturación, $C C$ = capacidad de campo, PMP = punto de marchitez permanente, $C H$ = conductividad hidráulica, $D A=$ densidad aparente, $C P=$ componente principal.

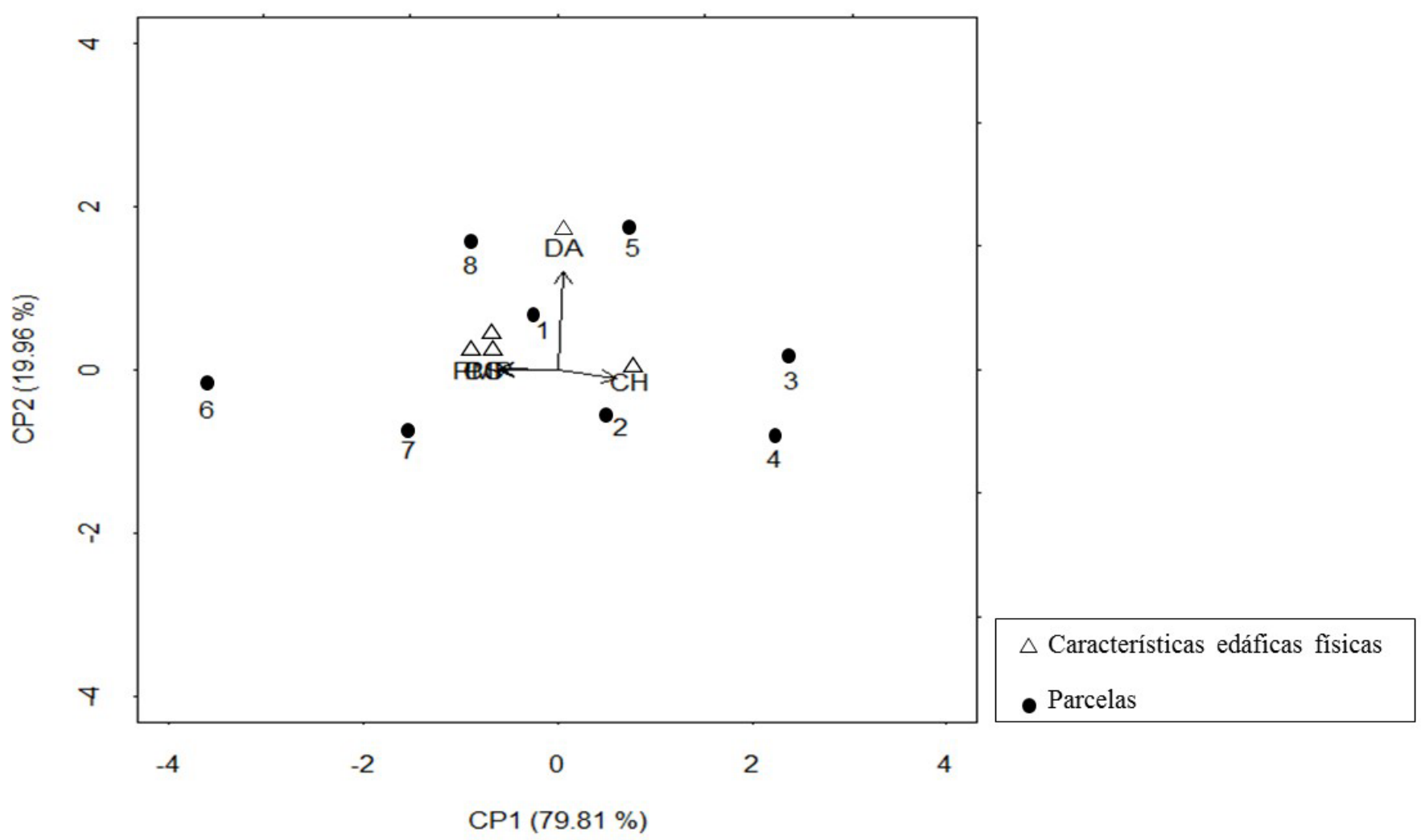

FIGURA 2. Correlación de los componentes principales 1 y 2 de las variables edáficas físicas analizadas en la plantación de G. arborea establecida en el Estado de México. 
TABLA 7. Autovalor, porcentaje de varianza y porcentaje de varianza acumulado asociado del análisis de componentes principales de las variables edáficas químicas analizadas en la plantación de G. arborea en Tlatlaya, Estado de México.

\begin{tabular}{cccc}
\hline Componente principal & Autovalor & Porcentaje de varianza & Porcentaje de varianza acumulado \\
\hline 1 & 3.4203 & 44.99 & 44.99 \\
2 & 2.2138 & 18.85 & 63.84 \\
3 & 1.9928 & 15.27 & 79.12 \\
4 & 1.6000 & 9.84 & 88.96 \\
5 & 1.2103 & 5.63 & 94.59 \\
6 & 0.9546 & 3.50 & 98.10 \\
\hline
\end{tabular}

TABLA 8. Coeficientes seleccionados y los primeros seis componentes principales del ACP de las variables edáficas químicas de la plantación de G. arborea en Tlatlaya, Estado de México.

\begin{tabular}{|c|c|c|c|c|c|c|}
\hline Variable & $\mathrm{CP1}$ & $\mathrm{CP} 2$ & CP3 & CP4 & CP5 & CP6 \\
\hline $\mathrm{MO}$ & 0.2341 & 0.2023 & 0.0692 & -0.1978 & 0.0774 & -0.1471 \\
\hline$P$ & 0.1599 & 0.3233 & 0.0983 & 0.0914 & 0.2790 & -0.0789 \\
\hline K & 0.1522 & 0.3607 & 0.1397 & 0.0647 & -0.0401 & -0.0033 \\
\hline $\mathrm{Ca}$ & -0.2500 & 0.2163 & -0.0193 & 0.0956 & -0.0983 & 0.0149 \\
\hline $\mathrm{Mg}$ & -0.2030 & 0.0147 & 0.2997 & -0.0316 & -0.2750 & 0.2063 \\
\hline $\mathrm{Na}$ & -0.2650 & 0.1778 & -0.0380 & 0.0725 & 0.0333 & 0.0496 \\
\hline $\mathrm{Fe}$ & 0.1497 & 0.1204 & 0.3434 & 0.1053 & 0.3300 & 0.0751 \\
\hline $\mathrm{Zn}$ & 0.1713 & 0.1574 & -0.1760 & -0.3212 & -0.3104 & 0.0400 \\
\hline $\mathrm{Mn}$ & 0.0365 & -0.3930 & 0.0081 & 0.0728 & 0.3040 & 0.2060 \\
\hline $\mathrm{Cu}$ & 0.0855 & -0.1512 & -0.2193 & -0.3204 & -0.0889 & -0.6007 \\
\hline B & 0.1578 & -0.0354 & 0.3677 & 0.0343 & -0.1132 & -0.3794 \\
\hline Al & 0.1146 & -0.2956 & 0.0340 & 0.3680 & 0.0401 & -0.2613 \\
\hline $\mathrm{S}$ & -0.1389 & -0.0437 & 0.0763 & 0.2915 & -0.3883 & -0.3040 \\
\hline N-NO3 & -0.0198 & -0.1877 & 0.0653 & -0.5564 & 0.0477 & 0.0607 \\
\hline $\mathrm{pH}$ & -0.2306 & 0.2160 & -0.1423 & 0.0241 & -0.2135 & 0.0132 \\
\hline $\mathrm{CT}$ & -0.2164 & 0.1984 & -0.0720 & 0.2045 & 0.0192 & -0.2175 \\
\hline Salin & 0.1529 & 0.0879 & 0.3548 & -0.1390 & -0.2819 & 0.0644 \\
\hline $\mathrm{Ca} / \mathrm{K}$ & -0.2867 & 0.0403 & -0.0268 & 0.0004 & 0.1087 & -0.1040 \\
\hline $\mathrm{Mg} / \mathrm{K}$ & -0.2160 & -0.1436 & 0.2782 & -0.1158 & -0.0555 & 0.0534 \\
\hline $\mathrm{Ca}+\mathrm{Mg} / \mathrm{K}$ & -0.2879 & -0.0120 & 0.0630 & -0.0342 & 0.0680 & -0.0646 \\
\hline $\mathrm{Ca} / \mathrm{Mg}$ & 0.0461 & 0.2524 & -0.3911 & -0.0357 & -0.0052 & 0.1728 \\
\hline $\mathrm{ClCa}$ & -0.2518 & 0.0818 & 0.0282 & -0.1710 & 0.2592 & -0.2025 \\
\hline $\mathrm{CiMg}$ & -0.2119 & -0.0286 & 0.3118 & -0.1326 & -0.1309 & 0.1155 \\
\hline CiK & 0.1489 & 0.3316 & 0.1845 & -0.0733 & 0.1532 & -0.1192 \\
\hline $\mathrm{CiNa}$ & -0.2605 & 0.0587 & -0.0012 & -0.1558 & 0.2626 & -0.1274 \\
\hline $\mathrm{CIC}$ & -0.2616 & 0.0481 & 0.1101 & -0.1584 & 0.1817 & -0.1534 \\
\hline
\end{tabular}

${ }^{*} \mathrm{CP}=$ componente principal. 
En la figura 3 se observa que el CP1 proporcionó mayor peso a las variables edáficas químicas $\mathrm{Ca}+\mathrm{Mg} / \mathrm{K}, \mathrm{Ca} / \mathrm{K}$, $\mathrm{Na}, \mathrm{CIC}$, CiNa y CICA en orden de importancia, se infiere que las parcelas 1, 2, 6 y 7 están relacionadas con las variables antes mencionadas por presentar los resultados mayores obtenidos en laboratorio. El CP2 proporcionó mayor peso a las variables edáficas químicas $\mathrm{Mn}, \mathrm{K}, \mathrm{CiK}, \mathrm{P}$ y Al, las cuales están relacionadas con las parcelas 5 y 8 pues presentan resultados mínimos y máximos de concentraciones.

Para el conjunto de variables topográficas estudiadas, se constituyeron tres componentes principales, que se muestran en la tabla 9. Se seleccionaron las variables pendiente y altitud por ser representativas de los componentes principales (Tabla 10).

En la figura 4 se observa que el CP1 proporcionó mayor peso a las variables topográficas profundidad y pendiente en orden de importancia; se infiere que las parcelas 3, 4, 5, 6, 7 y 8 están relacionadas con las variables antes mencionadas debido a que presentan valores máximos y mínimos obtenidos en campo. El CP2 proporcionó mayor peso a la variable topográfica altitud, que está relacionada con las parcelas 1 y 2 pues muestran la altitud máxima y mínima respectivamente.

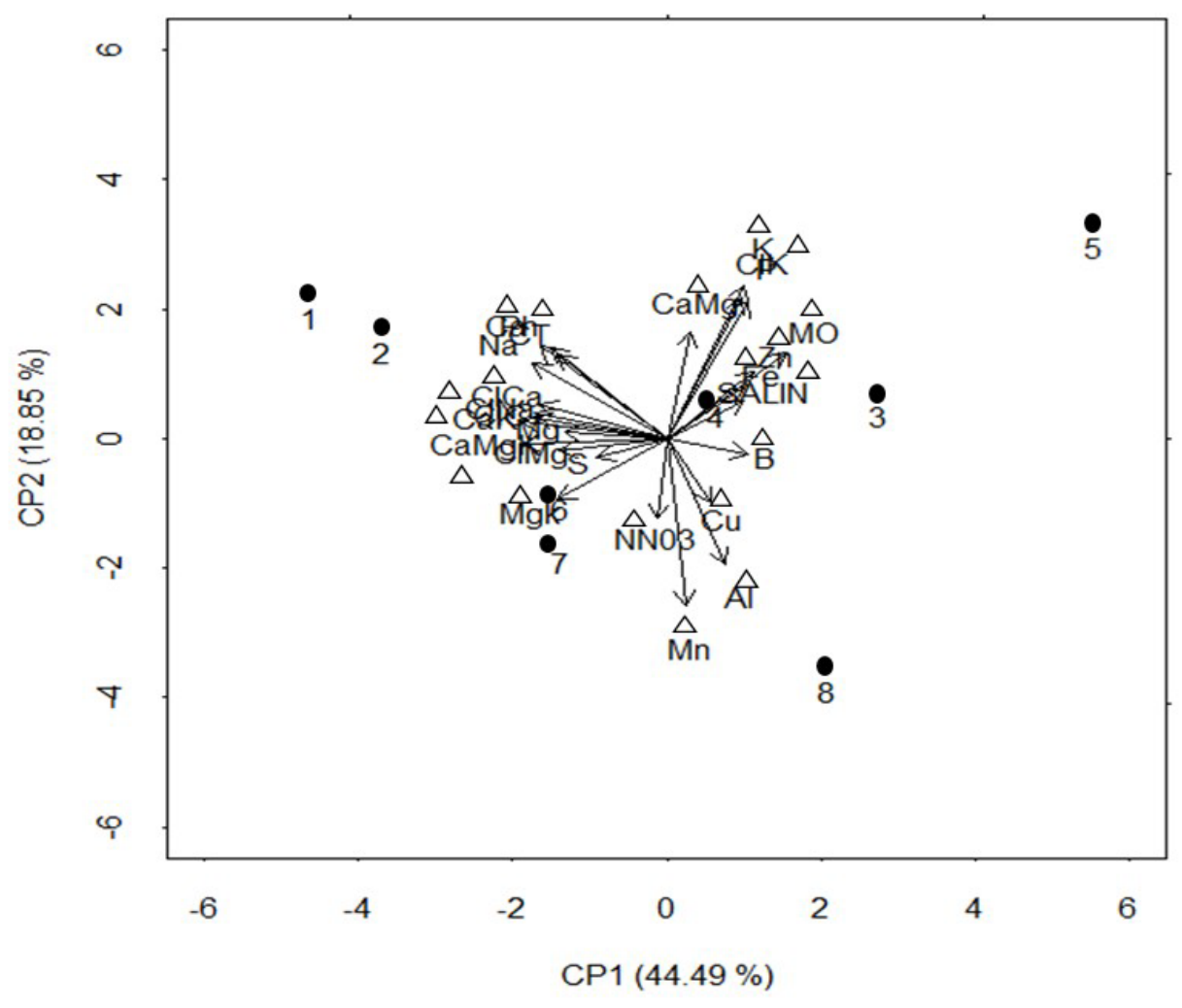

$\triangle$ Características edáficas químicas

- Parcelas

FIGURA 3. Correlación de los componentes principales de las variables edáficas químicas analizadas en la plantación de G. arborea establecida en el Estado de México. 
TABLA 9. Autovalor, porcentaje de varianza y porcentaje de varianza acumulado asociado del análisis de componentes principales de las variables topográficas analizadas en la plantación de G. arborea en Tlatlaya, Estado de México.

\begin{tabular}{cccc}
\hline Componente principal & Autovalor & Porcentaje de varianza & Porcentaje de varianza acumulado \\
\hline 1 & 1.2911 & 55.56 & 55.56 \\
2 & 1.0649 & 37.80 & 93.36 \\
3 & 0.4461 & 6.63 & 100.00 \\
\hline
\end{tabular}

TABLA 10. Coeficientes seleccionados y los primeros tres componentes principales del ACP de las variables topográficas de la plantación de G. arborea en Tlatlaya, Estado de México.

\begin{tabular}{cccc}
\hline Variables & CP1 & CP2 & CP3 \\
\hline Altit. & 0.0181 & 0.9252 & -0.3789 \\
Pend. & 0.7101 & 0.2549 & 0.6562 \\
Prof. & -0.7038 & 0.2809 & 0.6524 \\
\hline
\end{tabular}

Altit. $=$ altitud $(\mathrm{msnm})$, Pend $=$ pendiente $\left({ }^{\circ}\right)$, Prof. $=$ profundidad $(\mathrm{cm}), \mathrm{CP}=$ componente principal.

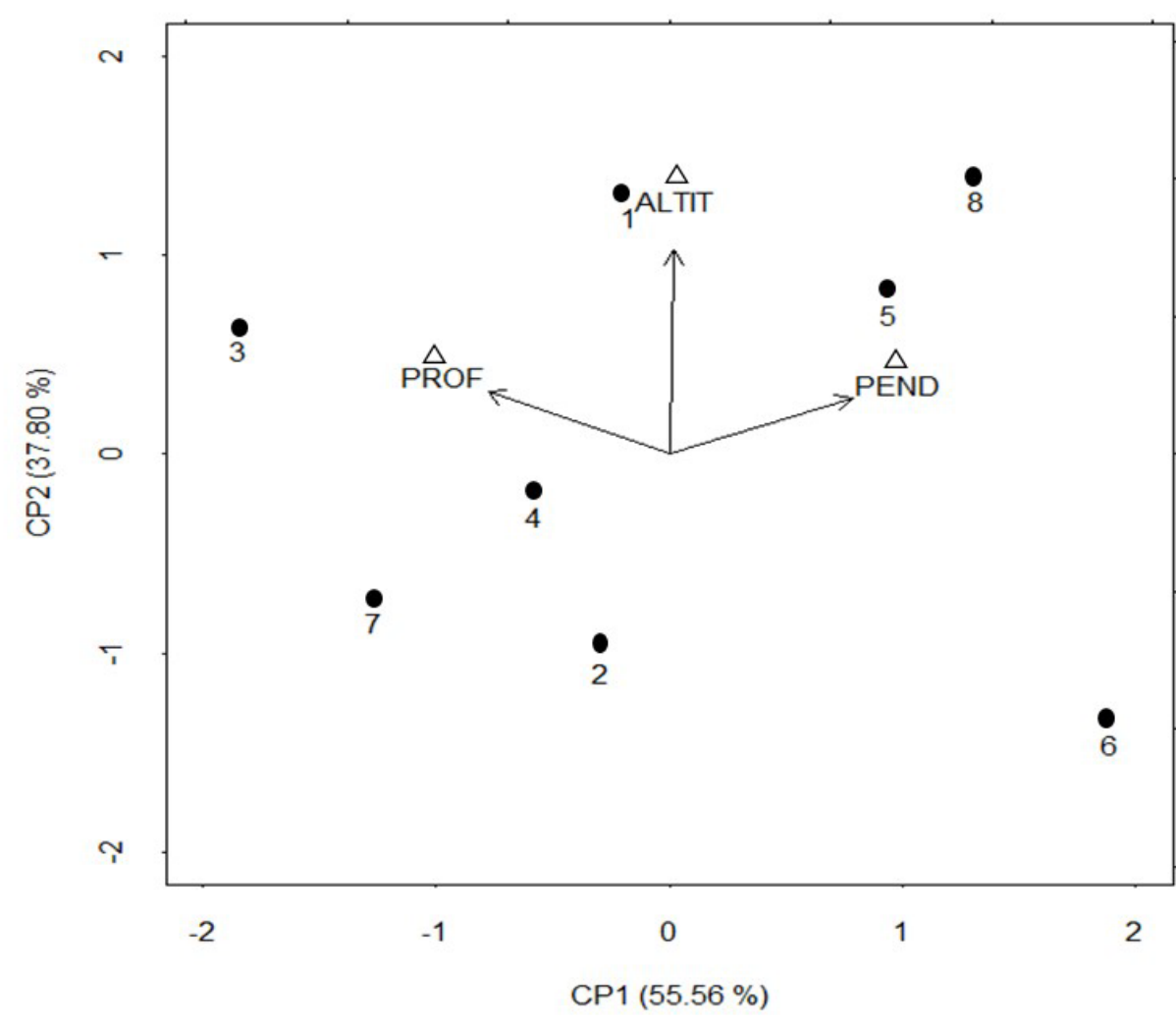

$\triangle$ Características topográficas

- Parcelas

FiguRA 4. Correlación de los componentes principales 1 y 2 de las variables topográficas analizadas en la plantación de G. arborea establecida en el Estado de México. 


\section{Análisis de regresión lineal múltiple}

Realizado el ACP sobre los tres conjuntos de características estudiadas, se seleccionaron 11 variables: $\mathrm{CC}, \mathrm{DA}, \mathrm{CH}, \mathrm{Ca}$ $+\mathrm{Mg} / \mathrm{K}, \mathrm{Mn}, \mathrm{Ca} / \mathrm{Mg}, \mathrm{N}-\mathrm{NO} 3, \mathrm{~S}, \mathrm{Cu}$, altitud y pendiente. La prueba de Shapiro-Wilk indicó que las variables presentaban normalidad a un nivel de confianza del $95 \%$, a excepción de la variable S la cual presentó p-valor $=0.004$.

En la tabla 11 se expone la matriz de correlaciones para las 10 variables independientes que fueron seleccionadas con base en el ACP y la variable volumen fustal, estas correlaciones muestran algunas similitudes positivas y significativas. Algunas expresan semejanza, aunque no significativa pero apreciable entre la CC y el volumen fustal.
Las variables introducidas como predictores en el análisis de regresión lineal múltiple se ordenaron de forma jerárquica, es decir de mayor a menor por su valor absoluto obtenido en la correlación. El resultado del análisis de regresión múltiple presentó un valor de $\mathrm{R}^{2}$ ajustado $=0.71$, lo que significa que este modelo es capaz de explicar $71.6 \%$ de la variabilidad observada en el volumen fustal de $G$. arborea, la prueba de $F$ muestra una significancia de $\mathrm{P} \leq$ 0.0466. Para este tipo de regresión múltiple se satisfacen todas las condiciones; por ello, se considera apropiado para estimar con mayor precisión el volumen fustal en función de las variables $\mathrm{CH}, \mathrm{N}-\mathrm{NO} 3$ y la relación $\mathrm{Ca} / \mathrm{Mg}$ (Tabla 12).

TABLA 11. Coeficientes de correlación entre volumen y las 10 variables independientes seleccionadas del ACP mediante el coeficiente de correlación de Pearson.

\begin{tabular}{|c|c|c|c|c|c|c|c|c|c|c|c|}
\hline & ${ }^{*} \mathrm{~V}$ & $\mathrm{CC}$ & $\mathrm{DA}$ & $\mathrm{CH}$ & $\mathrm{Ca}+\mathrm{Mg} / \mathrm{K}$ & $\mathrm{Mn}$ & $\mathrm{Ca} / \mathrm{Mg}$ & $\mathrm{N}-\mathrm{NO3}$ & $\mathrm{Cu}$ & Altit & Pend \\
\hline V & ${ }_{* *}^{1.00}$ & & & & & & & & & & \\
\hline CC & $\begin{array}{l}0.68 \\
n s\end{array}$ & 1.00 & & & & & & & & & \\
\hline DA & $\begin{array}{l}-0.54 \\
\text { ns }\end{array}$ & $\begin{array}{l}-0.10 \\
n s\end{array}$ & ${ }_{* *}^{1.00}$ & & & & & & & & \\
\hline $\mathrm{CH}$ & $\begin{array}{l}-0.64 \\
n s\end{array}$ & -0.99 & $\begin{array}{l}\text { O.01 } \\
\text { ns }\end{array}$ & 1.00 & & & & & & & \\
\hline $\mathrm{Ca}+\mathrm{Mg} / \mathrm{K}$ & $\begin{array}{l}0.62 \\
\text { ns }\end{array}$ & $\begin{array}{l}0.42 \\
\mathrm{~ns}\end{array}$ & $\begin{array}{l}-0.55 \\
\text { ns }\end{array}$ & $\begin{array}{l}-0.42 \\
n s\end{array}$ & ${ }_{* *}^{1.00}$ & & & & & & \\
\hline $\mathrm{Mn}$ & $\begin{array}{l}-0.18 \\
\text { ns }\end{array}$ & $\begin{array}{l}0.36 \\
\text { ns }\end{array}$ & $\begin{array}{l}-0.12 \\
\mathrm{~ns}\end{array}$ & $\begin{array}{l}-0.30 \\
n s\end{array}$ & $\begin{array}{l}-0.09 \\
\text { ns }\end{array}$ & $\underbrace{1.00}_{* *}$ & & & & & \\
\hline $\mathrm{Ca} / \mathrm{Mg}$ & $\begin{array}{l}-0.50 \\
n s\end{array}$ & -0.94 & $\begin{array}{l}-0.02 \\
n s\end{array}$ & 0.96 & $\begin{array}{l}-0.28 \\
n s\end{array}$ & $\begin{array}{l}-0.43 \\
\mathrm{~ns}\end{array}$ & $\underbrace{1.00}_{* *}$ & & & & \\
\hline $\mathrm{N}-\mathrm{NO3}$ & $\begin{array}{l}0.60 \\
n s\end{array}$ & $\begin{array}{l}0.34 \\
\mathrm{~ns}\end{array}$ & $\begin{array}{l}-0.66 \\
\mathrm{~ns}\end{array}$ & $\begin{array}{l}-0.23 \\
n s\end{array}$ & $\begin{array}{l}0.14 \\
\text { ns }\end{array}$ & $\begin{array}{l}0.30 \\
\mathrm{~ns}\end{array}$ & $\begin{array}{l}-0.27 \\
n s\end{array}$ & $\underbrace{1.00}_{* *}$ & & & \\
\hline $\mathrm{Cu}$ & $\begin{array}{l}-0.11 \\
\text { Ns }\end{array}$ & $\begin{array}{l}-0.22 \\
n s\end{array}$ & $\begin{array}{l}0.07 \\
\text { ns }\end{array}$ & $\begin{array}{l}0.26 \\
\text { ns }\end{array}$ & $\begin{array}{l}-0.28 \\
n s\end{array}$ & $\begin{array}{l}0.09 \\
\text { ns }\end{array}$ & $\begin{array}{l}0.12 \\
\text { ns }\end{array}$ & $\begin{array}{l}0.47 \\
\text { ns }\end{array}$ & ${ }_{* *}^{1.00}$ & & \\
\hline Altit & $\begin{array}{l}-0.53 \\
n s\end{array}$ & $\begin{array}{l}-0.25 \\
n s\end{array}$ & 0.80 & $\begin{array}{l}0.22 \\
\text { ns }\end{array}$ & $\begin{array}{l}-0.33 \\
n s\end{array}$ & $\begin{array}{l}-0.05 \\
\text { ns }\end{array}$ & $\begin{array}{l}0.26 \\
\text { ns }\end{array}$ & $\begin{array}{l}-0.52 \\
\text { ns }\end{array}$ & $\begin{array}{l}0.28 \\
\text { ns }\end{array}$ & $\underbrace{1.00}_{* \star}$ & \\
\hline Pend & $\begin{array}{l}-0.28 \\
n s\end{array}$ & $\begin{array}{l}0.31 \\
\text { ns }\end{array}$ & $\begin{array}{l}0.63 \\
\mathrm{~ns}\end{array}$ & $\begin{array}{l}-0.37 \\
n s\end{array}$ & $\begin{array}{l}-0.36 \\
n s\end{array}$ & $\begin{array}{l}0.17 \\
\mathrm{~ns}\end{array}$ & $\begin{array}{l}-0.39 \\
n s\end{array}$ & $\begin{array}{l}-0.59 \\
\text { ns }\end{array}$ & $\begin{array}{l}-0.48 \\
\text { ns }\end{array}$ & $\begin{array}{l}0.24 \\
\text { ns }\end{array}$ & $\begin{array}{l}1.00 \\
\text { ns }\end{array}$ \\
\hline
\end{tabular}

Se marca en negritas las variables con los mayores valores de correlación. 
TABLA 12. Valores de los estadísticos de bondad de ajuste del modelo evaluado en la estimación del volumen fustal de G. arborea.

\begin{tabular}{cccccc}
\hline Coeficientes & Valor estimado & Error Estándar & t value & Pr>t & $R^{2}$ ajustado \\
\hline Intercepto & 0.019070 & 0.006847 & 2.785 & 0.0496 & \\
$\mathrm{CH}$ & -0.006113 & 0.002062 & -2.965 & 0.0414 & 0.716 \\
$\mathrm{NNO3}$ & 0.002902 & 0.001083 & 2.679 & 0.0463 & \\
$\mathrm{CaMg}$ & 0.006101 & 0.002647 & 2.305 & 0.0452 & \\
\hline
\end{tabular}

$R^{2}$ adj = coeficiente de determinación ajustado.

TABLA 13. Resultados de las pruebas los supuestos de la regresión.

\begin{tabular}{|c|c|c|c|c|c|}
\hline Modelo & $\begin{array}{l}\text { Shapiro- } \\
\text { Wilk (W) }\end{array}$ & $P r<W$ & $\begin{array}{l}\text { Durbin-Watson } \\
\text { (DW) }\end{array}$ & $\begin{array}{l}\text { Breusch- } \\
\text { Pagan (BP) }\end{array}$ & Pr>Chi-Sq \\
\hline $\begin{array}{r}V=0.019070+-0.006113 * C H+0.002902 \\
* N N 03+0.006101 * C a M g\end{array}$ & 0.96503 & 0.8564 & 1.5684 & 3.3615 & 0.3392 \\
\hline
\end{tabular}

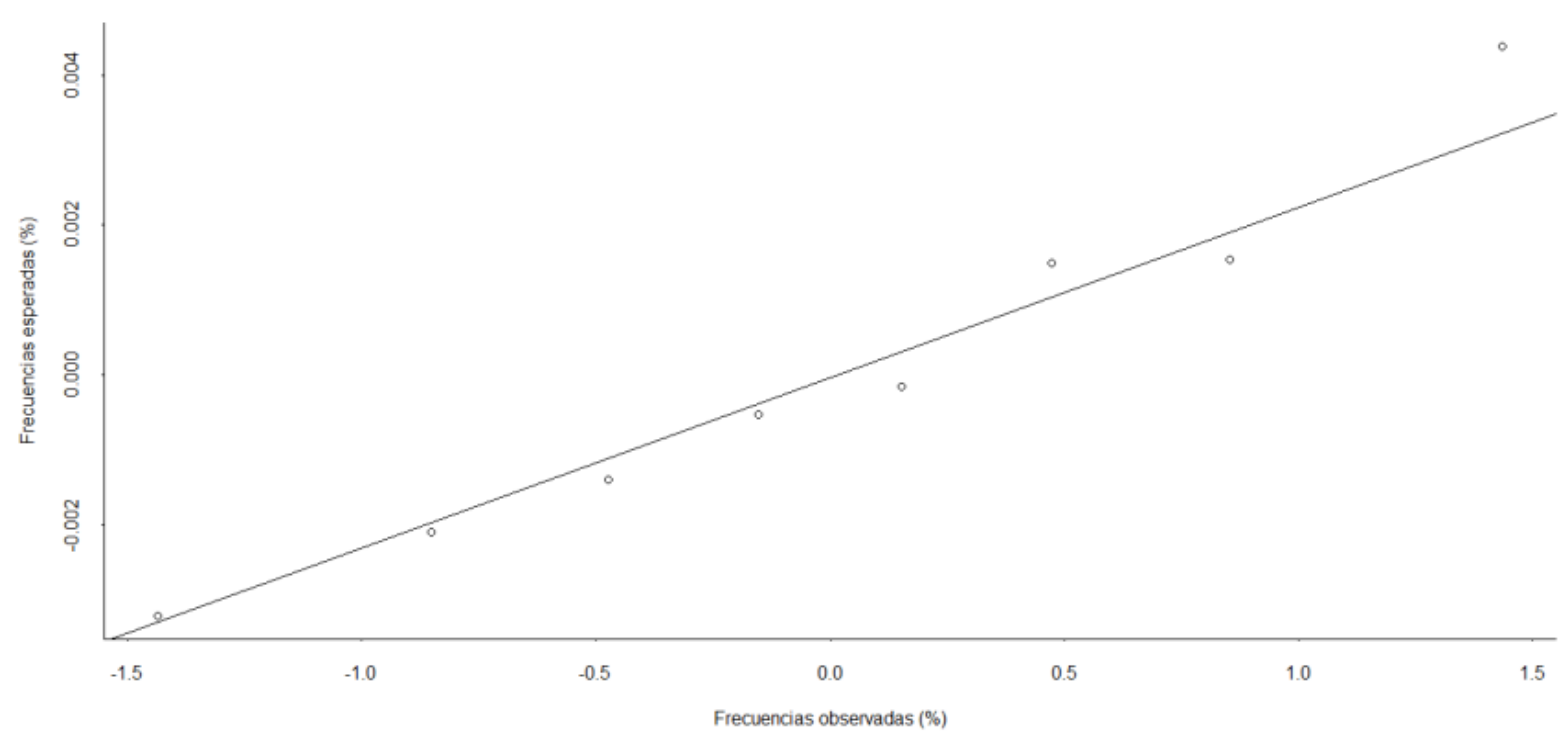

FIgURA 5. Porcentajes acumulados de los residuales frente a la distribución normal para el modelo ajustado para la plantación de G. arborea establecida en el Estado de México.

Una vez examinado el cumplimiento de los supuestos de la regresión en el modelo, la prueba de normalidad de ShapiroWilk, mostró un valor de 0.96 (Tabla 13). Los porcentajes de frecuencias relativas acumuladas de los residuales muestran una relación cercana a una línea recta (Fig. 5). Respecto a la probabilidad de la distribución normal, los porcentajes tienden a formar una campana de Gauss. La dispersión de los residuos, respecto a los valores estimados de volumen del modelo (Fig. 6) no presenta evidencia de alguna deficiencia. La prueba de Breusch-Pagan obtuvo un valor de 3.36 (Tabla 13), el cual no es significativo en los residuales $(\operatorname{Pr}>$ ChiSq $=0.3392)$, no existe evidencia estadística significativa de heterocedasticidad. El estadístico Durbin-Watson de independencia de la frecuencia de los residuos demuestra que no hay colinealidad entre variables (Tabla 13). 

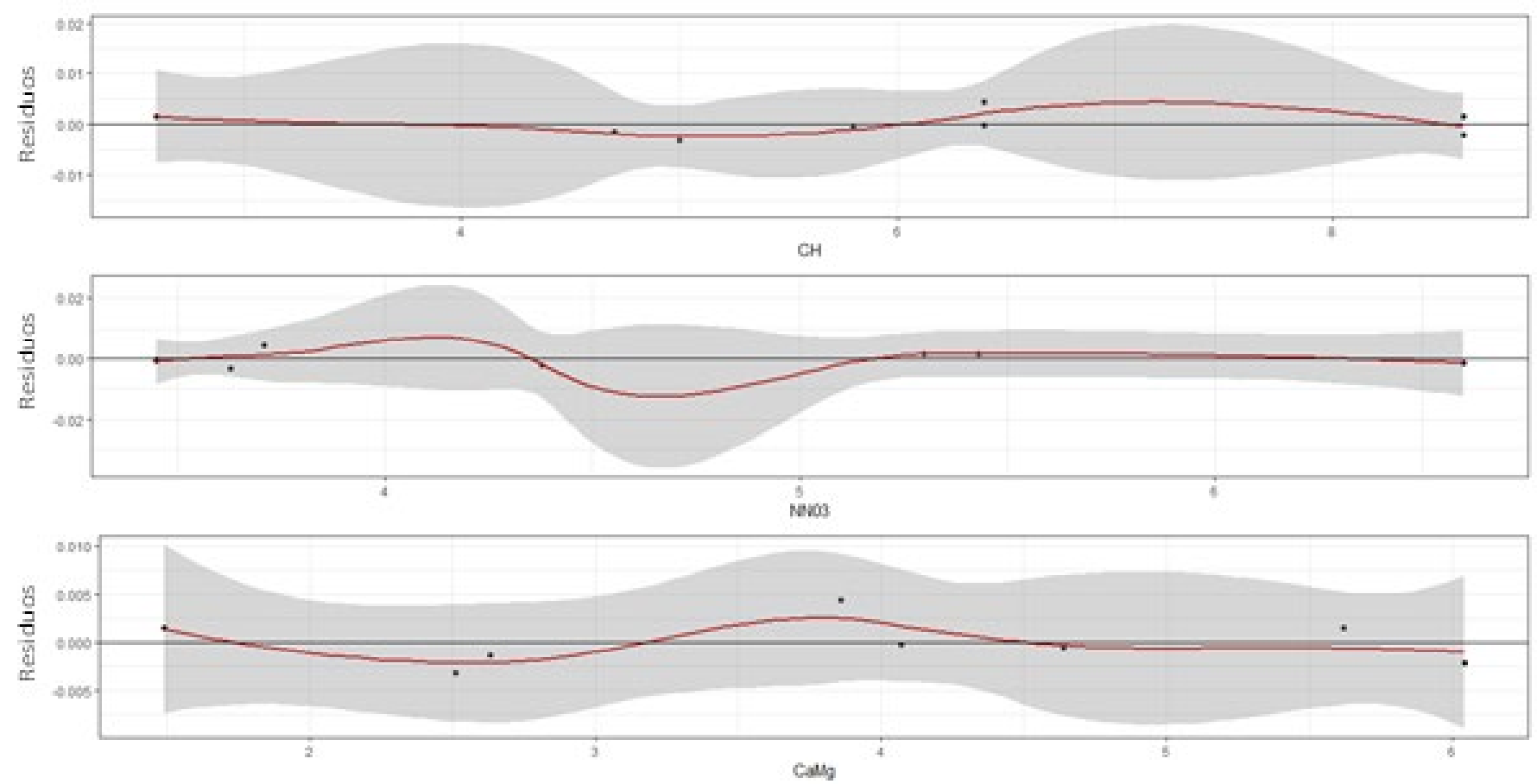

FIgura 6. Distribución de residuos del modelo ajustado de volumen fustal de la plantación de G. arborea establecida en el Estado de México.

Del proceso de selección dentro del análisis de regresión lineal múltiple, el mejor modelo resultante para la estimación del volumen fustal queda definido como:

$$
\begin{array}{r}
V=0.019070+-0.006113 * C H+0.002902 \\
* N N 03+0.006101 * C a M g
\end{array}
$$

Donde:

$V=\operatorname{Volumen}$ fustal $\left(\mathrm{m}^{3}\right)$

$\mathrm{CH}=$ Conductividad Hidráulica $\left(\mathrm{cm} \mathrm{h}^{-1}\right)$

NNO3 $=$ Nitratos de Nitrógeno $(\mathrm{ppm})$

$\mathrm{CaMg}=$ Relación Calcio/Magnesio (meq/100 g)

\section{DISCUSIÓN}

\section{Características edáficas físicas}

Autores como Alfaro, de Camino y Varmola (2002) y Martinez (2015) mencionan que altos porcentajes de arcilla se constituyen como uno de los principales limitantes en el desarrollo de G. arborea, mientras que, Rojas y Murillo (2004) recomiendan establecer plantaciones sobre suelos con texturas francas, franca arcillosa y bien aireadas. Los suelos de las parcelas de estudio donde se desarrolla $G$. arborea presentaron diferente textura (francosa, francoarcillosa y franco arcillo-arenosa); esta característica tiene importancia en el crecimiento forestal, determina en gran medida la capacidad de retención de agua y nutrientes, así como la aireación del suelo (Martínez et al., 2008).

La densidad aparente por su parte suele variar entre 0.2 $\mathrm{g} \mathrm{cm}^{-3}$ en las capas orgánicas y $1.92 \mathrm{~g} \mathrm{~cm}^{-3}$ en arenas gruesas (Binkley y Fisher, 1999); de esta manera la porosidad media de los suelos forestales varía entre 35\% y 65\% (Pritchett, 1986); por lo tanto, se trata de suelos compactos o poco porosos, lo cual puede provocar anegamientos, en consecuencia el crecimiento de G. arborea se reduce cuando los suelos están compactados ( $\mathrm{DA}>0.9 \mathrm{~g} \mathrm{~m}^{3}$ ) (Martínez, 2015). Si las condiciones del suelo son adversas, sus individuos crecen de manera defectuosa: fustes torcidos, 
poca altura, muy ramificados y aspecto arbustivo (RuizBlandon et al., 2020); lo anterior concuerda con los valores que aquí se determinaron para la DA que presentó un valor promedio de $1.26 \mathrm{~g} \mathrm{~cm}^{-3}$.

La conductividad hidráulica se encuentra en el intervalo observado por Mariño-Macana y Rodríguez-Melo (2010), en una plantación clonal de G. arborea establecida en Zambrano, Colombia a una altitud de 20 m s.n.m., dichos autores obtuvieron una categoría de $0.05 \mathrm{~cm} \mathrm{~h}^{-1}$ a $6.10 \mathrm{~cm}$ $\mathrm{h}^{-1}$. La capacidad de campo es una medida útil para calcular dosis de riego de los cultivos y da una idea real de las características hídricas del suelo (Garrido, 1971), se trata de suelos con CC de nivel medio bajo $(12 \%-20 \%)$.

\section{Características edáficas químicas}

Adekunle, Alo y Adekayode (2011) evaluaron suelos que van de neutros a alcalinos ( $\mathrm{pH}$ entre 6.47 y 7.47) en plantaciones de G. arborea en Nigeria; Adeboye et al. (2011) obtuvieron un valor de $\mathrm{pH}$ de 6.9 en profundidades de 5 $\mathrm{cm}-10 \mathrm{~cm}$ en plantaciones de G. arborea establecidas en Nigeria, el resultado es superior a lo obtenido en la plantación de estudio. Kumar et al. (2011) obtuvo un valor de $\mathrm{pH}$ de 6.67 a una profundidad de $20 \mathrm{~cm}$ a $30 \mathrm{~cm}$ en el bosque seco tropical en India donde crece de forma natural G. arborea; Barrios et al. (2011) obtuvieron resultados de $\mathrm{pH}$ de 6.9 que es moderadamente alcalino en una plantación de G. arborea de 4 años de edad establecida en Tolima, Colombia a 317 m s.n.m., sobre suelos de origen aluvial de textura franco arenosos a profundidades de $0 \mathrm{~cm}-30 \mathrm{~cm}$ y con una pendiente de $7^{\circ}$.

Se ha registrado que el intervalo de $\mathrm{pH}$ del suelo en el cual se ven favorecidos el crecimiento en diámetro normal, la altura total y el volumen total es entre 5 y 6.4 (Vásquez y Ugalde, 1996; Vallejos, 1996; Rojas y Murillo, 2004); por su parte, Martínez (2015) demuestra que altos crecimientos se procuran en suelos con $\mathrm{pH}$ arriba de 6.0, G. arborea crece vigorosamente en suelos con capas superficiales alcalinas o ligeramente ácidas, donde encuentran una amplia gama de nutrimentos disponibles, no obstante, fracasará en suelos ácidos muy lixiviados donde puede prosperar el género Pinus.
La salinidad obtenida en las parcelas de estudio es inferior a lo observado por Salcedo-Pérez et al. (2014) sobre una plantación de $T$. grandis establecida en Nayarit de dos años de edad sobre suelos de textura arenosa con $\mathrm{pH}$ que oscila de 5.3 a 6.1. Estos autores obtuvieron valores que fluctúan entre $0.51 \mathrm{dS} \mathrm{m}^{-1}$ y $0.59 \mathrm{dS} \mathrm{m}^{-1}$, lo cual indica que es una variable edáfica de suma importancia que se relaciona con la absorción de nutrimentos y agua; por su parte, Garrido (1971) muestra que los cultivos presentan un

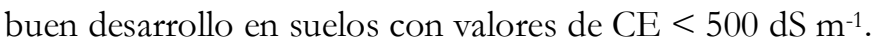

El resultado de MO es afín a lo hallado por Adekunle et al. (2011), quienes determinaron un contenido de MO de $2.65 \%$ en una plantación de G. arborea de 14 años de edad en Nigeria e inferior a lo obtenido por Ruiz-Blandon et al. (2020) en plantaciones de G. arborea establecidas en Nayarit, estos últimos autores registraron un contenido de MO de $3.2 \%$ en suelos con textura franco-arcillosa; Garrido (1971) recomienda elevar el contenido de $\mathrm{MO}$ a 3\% cuando la productividad es baja o existe riesgo de erosión.

$\mathrm{El}$ resultado de $\mathrm{P}$ de las parcelas de estudio difiere con Salcedo-Pérez et al. (2014), quienes obtuvieron valores de $18 \mathrm{ppm}$ a $42 \mathrm{ppm}$ en profundidades de $40 \mathrm{~cm}$ en plantaciones de T. grandis de dos años de edad establecidas en Nayarit. Se alude que este elemento repercute en el crecimiento de los árboles específicamente sobre el desarrollo de las raíces (Garrido, 1971). Espinoza, Slaton y Mozaffari (2012) exponen que concentraciones menores de 16 ppm de P en suelos, el potencial de crecimiento es de $65 \%$.

El resultado de $\mathrm{K}$ de las parcelas de estudio se encuentra dentro del intervalo 91 ppm - 130 ppm, de acuerdo con Espinoza et al. (2012), esas concentraciones de $\mathrm{K}$ en el suelo el potencial de crecimiento es de $85 \%$ a $95 \%$. Con respecto al Ca, concentraciones $\leq 400 \mathrm{ppm}$ en el suelo corresponden a un nivel bajo, por esta razón, el potencial de crecimiento es de 65\% a 85\% (Espinoza et al., 2012); Espinoza et al. (2012) determinaron que concentraciones $\leq$ $30 \mathrm{ppm}$ de $\mathrm{Mg}$ en el suelo representan a un nivel bajo, el potencial de crecimiento es de $65 \%$ a $85 \%$. Con relación al elemento $\mathrm{Fe}$, el resultado obtenido en las parcelas de 
estudio es alto, al aumentar la acidez del suelo, se incrementa la actividad del $\mathrm{Fe}$, que puede resultar tóxico.

Concentraciones $\leq 1.6 \mathrm{ppm}$ de $\mathrm{Zn}$ en el suelo son de nivel bajo, así pues, el potencial de crecimiento es $<65 \%$ (Espinoza et al., 2012). Respecto al elemento Cu, Barrios et al. (2011) observaron 0.20 ppm de este elemento; Espinoza et al. (2012) refieren que concentraciones $\leq 1.0 \mathrm{ppm}$ de $\mathrm{Cu}$ en el suelo es de nivel bajo, por ende el potencial de crecimiento es de $65 \%$ a $85 \%$.

Barrios et al. (2011) obtuvieron un contenido de Mn de 14.00 ppm; Espinoza et al. (2012) expresan que concentraciones $\leq 40 \mathrm{ppm}$ de $\mathrm{Mn}$ en el suelo es de nivel bajo, de manera que el potencial de crecimiento es de $65 \%$ a $85 \%$. Con respecto al B, Barrios et al. (2011) mencionan un promedio de $1.0 \mathrm{ppm}$. El N aprovechable por las plantas es en forma de N-NO3 depende de la cantidad de nitrógeno amoniacal presente, ya sea que provenga de $\mathrm{MO}$ o de sales inorgánicas de amonio (Garrido, 1971).

La CIC definida como la capacidad del suelo para retener e intercambiar diferentes elementos minerales, aumenta proporcionalmente a la cantidad de $\mathrm{MO}$, la cual es la base en la fertilidad del suelo (Fernández y Rojas, 2006), de acuerdo con Garrido (1971) se trata de suelos con nivel bajo (10 meq/100 g - $20 \mathrm{meq} / 100 \mathrm{~g}$ ) que requieren aporte de MO.

En relación con los cationes intercambiables de $\mathrm{Ca}$, Adeboye et al. (2011) obtuvieron un valor de 2.80 $\mathrm{meq} / 100 \mathrm{~g}$ a profundidades de $5 \mathrm{~cm}-10 \mathrm{~cm}$ en plantaciones de G. arborea establecidas en Nigeria. Por su parte Barrios et al. (2011) determinaron un valor de $11 \mathrm{meq} / 100 \mathrm{~g}$; Rojas y Murillo (2004) recomiendan que los contenidos de Ca deben ser mayores de $10 \mathrm{meq} / 100 \mathrm{~g}$, el intervalo del contenido de $\mathrm{Ca}$ en el que $G$. arborea crece normalmente oscila entre 6.0 y $22.3 \mathrm{meq} / 100 \mathrm{~g}$ (Vallejos, 1996), a medida que se pierde el calcio intercambiable del suelo por agotamiento aumenta gradualmente la acidez.

El resultado de $\mathrm{Mg}$ es superior a lo obtenido por Adeboye et al. (2011) en profundidades de $5 \mathrm{~cm}-10 \mathrm{~cm}$ en plantaciones de G. arborea establecidas en Nigeria (2.40 meq/100 g) y a lo observado por Barrios et al. (2011) (0.80 meq/100 g); Rojas y Murillo (2004) recomiendan que los contenidos de $\mathrm{Mg}$ deben ser mayores de 6 meq/100g. $G$. arborea crece normalmente cuando el contenido de $\mathrm{Mg}$ es de entre $1.6 \mathrm{meq} / 100 \mathrm{~g}$ y $6.7 \mathrm{meq} / 100 \mathrm{~g}$ (Vallejos, 1996), conforme se pierde el magnesio intercambiable del suelo por agotamiento aumenta gradualmente la acidez.

El resultado de $\mathrm{K}$ es mayor con respecto a lo observado por Barrios et al. (2011), quienes determinaron un valor de $0.11 \mathrm{meq} / 100 \mathrm{~g}$, y menor con respecto a lo obtenido por Adeboye et al. (2011), quienes hallaron un valor de $1.96 \mathrm{meq} / 100 \mathrm{~g}$ en profundidades de $5 \mathrm{~cm}-10 \mathrm{~cm}$. Esta especie crece normalmente en sitios donde el contenido de K oscila entre 0.5 y 0.7 meq $/ 100 \mathrm{~g}$ de acuerdo con Vallejos (1996).

Con respecto a la relación $\mathrm{Ca} / \mathrm{K}$ debe variar entre 15 me/100 g y 30 me/100 g (Gómez, 2005), por lo anterior, se deriva que existe deficiencia de $\mathrm{K}$ debido a que el resultado es $>30$. En la relación $\mathrm{Mg} / \mathrm{K}$, el valor ideal es de entre 10 me/100 g y 15 me 100g (Gómez, 2005), en el presente estudio el resultado se encuentra en un intervalo aceptable, además es similar a lo obtenido por Adekunle et al. (2011) quienes determinaron un valor de $12.32 \mathrm{me} 100 \mathrm{~g}$ en una plantación de G. arborea de 10 años de edad establecida en Nigeria.

Sobre la relación $\mathrm{Ca}+\mathrm{Mg} / \mathrm{K}$, el valor ideal es de 20 me 100 g y 40 me 100 g (Gómez, 2005), se infiere que existe deficiencia de $\mathrm{K}$ debido a que el resultado es $>40$. En la relación $\mathrm{Ca} / \mathrm{Mg}$, el valor ideal es de entre 3 me 100g y 6 me $100 \mathrm{~g}$ (Gómez, 2005); en las parcelas de estudio, ésta relación se encuentra en el intervalo ideal.

\section{Características topográficas}

Respecto a la distribución altitudinal G. arborea exhibe mayor crecimiento entre $0 \mathrm{~m}$ y $900 \mathrm{~m}$ s.n.m., como lo señalaron Rojas y Murillo (2004); de acuerdo con estos autores, las parcelas de este estudio se encuentran en este intervalo adecuado de altitud, aunque no muestran mejores crecimientos como lo señala Martínez (2015), quien indica que los mejores crecimientos se producen debajo de los 300 m s.n.m. Con respecto a la pendiente, Rojas y Murillo (2004) y Martínez (2015) indican que G. arborea demuestra 
un buen crecimiento en sitios no superiores a $16^{\circ}$, además señalan que las plantaciones de G. arborea no prosperan en suelos erosionados o compactados. En esas condiciones biofísicas los árboles pueden mostrar características como fustes inclinados, poca altura, ser ramificados y tener aspecto arbustivo, por esta razón, se sugiere plantar esta especie en suelos profundos, húmedos, pero bien drenados y sin obstáculos de desarrollo radical. Con base en lo anterior, Rojas y Murillo (2004) y Martínez (2015) sugieren que los mejores sitios para G. arborea se ubican en las partes bajas de los terrenos, donde por lo general tienen mayor disponibilidad de agua y nutrientes, y los sitios con buenos contenidos de calcio y magnesio y los ubicados en áreas donde el uso anterior eran cultivos agrícolas.

Ávila et al. (2015) establecieron una clasificación de sitios (I, II, III, IV) para plantaciones clonales de G. arborea establecidas en Costa Rica con base en parámetros edafoclimáticos de la zona Pacífico Sur. Considerando esa clasificación, las características edáficas y topográficas de este estudio, la ubican en las clases III y IV, pues la topografía es ondulada, la pendiente de $40 \%$, la precipitación es menor a $2000 \mathrm{~mm}_{\text {año }}{ }^{-1}$, la textura es franca arcillo arenosa, la capacidad de intercambio catiónico se encuentra en el intervalo $11 \%$ - 25\%; únicamente el $\mathrm{pH}$ (6.5) ubica al sitio en la clase I.

\section{Variables dasométricas}

Ávila et al. (2015) estudiaron la interacción genotipo-sitio de dos plantaciones clonales de G. arborea de dos años de edad, en el Pacífico Sur de Costa Rica en suelos de tipo inceptisoles, con una pendiente de $1^{\circ}$, altitud de $26 \mathrm{~m}$; el diámetro normal promedio de la procedencia zona sur fue de $15.87 \mathrm{~cm}$ y $17.08 \mathrm{~cm}$ y la procedencia zona norte mostró un diámetro normal de $17.20 \mathrm{~cm}$ y $16.10 \mathrm{~cm}$; estos resultados son superiores a los obtenidos en la plantación en estudio e indican que la productividad del sitio es el resultado de complejas interacciones que ocurren entre distintos factores, dentro de los que destacan los edafoclimáticos, prácticas silviculturales y la genética del árbol.
El coeficiente de variación del diámetro normal fue de $4 \%$ en la plantación de estudio que, de acuerdo con Murillo, Hernández y Murillo (1996), indica una plantación homogénea, pues tiene un coeficiente de variación menor a $10 \%$. Este resultado refleja que se han realizado podas oportunas conforme crecen los árboles.

\section{Análisis de regresión lineal múltiple}

Adekunle et al. (2011) determinaron correlaciones significativas negativas entre el contenido de $\mathrm{K}$, la relación $\mathrm{Mg} / \mathrm{K}$ y, en menor grado, el contenido de Ca con el volumen fustal en plantaciones de diferentes edades en Nigeria. Se deduce que la variación de volumen fustal está fuertemente asociada a algunas características físicas y químicas del suelo donde se encuentra establecida $G$. arborea. Esto concuerda con lo observado por Kumar et al. (2011), quienes determinaron que las diferencias de estructura y composición del bosque seco tropical donde crece $G$. arborea de forma natural están correlacionadas significativamente con la variación de las propiedades del suelo; estos mismos autores indican que las propiedades el suelo tienen una influencia sustancial en la composición del bosque subtropical.

Los resultados de $\mathrm{CH}$ obtenidos concuerdan con los de Mariño-Macana y Rodríguez-Melo, (2010) quienes generaron un modelo para establecer el PMP en plantaciones de G. arborea establecidas en Colombia a una altitud de $20 \mathrm{~m} \mathrm{~s} \mathrm{n} \mathrm{m}$, estos autores utilizaron ocho propiedades fisicoquímicas como variables predictoras entre las que destacan DA y DR.

\section{CONCLUSIONES}

La variación de las características físicas y químicas del suelo y las topográficas intervienen en el crecimiento del volumen fustal de la plantación de G. arborea, por lo tanto, es válido estudiar los factores ambientales que se asocian a este comportamiento. Las características edáficas conductividad hidráulica, nitratos y la relación de cationes intercambiables $\mathrm{Ca} / \mathrm{Mg}$ fueron las variables más preponderantes en la 
definición del modelo para estimar el volumen fustal de $G$. arborea. En este estudio no se incluyeron aspectos climáticos que, asociados con las características edáficas, explican en mayor proporción la variación del crecimiento de volumen fustal de G. arborea.

\section{REFERENCIAS}

Adeboye, M. K. A., Bala, A., Osunde, A. O., Uzoma, A. O., Odofin, A. J., \& Lawal, B. A. (2011). Assessment of soil quality using soil organic carbon and total nitrogen and microbial properties in tropical agroecosystems. Agricultural Sciences, 02(01), 34-40. doi: 10.4236/as.2011.21006

Adekunle, V. A. J., Alo, A. A., \& Adekayode, F. O. (2011). Yields and nutrient pools in soils cultivated with Tectona grandis and Gmelina arborea in Nigerian rainforest ecosystem. Journal of the Saudi Society of Agricultural Sciences, 10(2), 127-135. doi: 10.1016/j.jssas.2011.05.001

Alfaro, M., Camino, R. De, \& Varmola, M. (2002). Melina (Gmelina arborea) in central America. Recuperado de http://agris.fao.org/agris-

search/search.do? recordID=XF2016039440

Álvarez, J. G., Barrio, M., Diéguez, U., \& Rojo, A. (2003). Metodología para la construcción de curvas de calidad de estación. Cuadernos de La Sociedad Española de Ciencias Forestales, 309(1), 303-309. doi: $10.31167 /$ csef.v0i18.9475

Ávila, C. A., Murillo, R. C., Murillo, O. G., \& Sandoval, C. S. (2015). Interacción genotipo sitio para dos conjuntos clonales de Gmelina arborea Roxb., en sitios planos del Pacífico Sur de Costa Rica. Revista Forestal Mesoamericana Kurú, 12(29), 2-14. Recuperado de http://hdl.handle.net/2238/8035

Barrios, A., López, A. M., Nieto, V., Burgos, N., Yaya, M., \& González, I. (2011). Efecto del control de malezas y fertilización sobre el crecimiento inicial de Gmelina arborea Roxb. en el Departamento del Tolima, Colombia. Colombia Forestal, 14(1), 31-40. Recuperado de

https://revistas.udistrital.edu.co/index.php/colfor/article/view $/ 3548 / 5145$

Binkley, D., \& Fisher, R. F. (1999). Ecology and Management of Forest Soils. Wiley. doi: 10.1002/9781119455745

Bueis, T. M. (2012). Influencia de los parámetros edáficos, topográficos y climáticos sobre el índice de sitio de Pinus sylvestris L. en las masas de León y Palencia. Tesis de Maestría. Universidad de Valladolid. Valladolid, España. 31 pp. Recuperado de http://sostenible.palencia.uva.es/system/files/publicaciones/T FM_Teresa_de_los_Bueis.pdf
Comisión Nacional Forestal [Conafor] (2014). Principales especies maderables en PFC por entidad federativa en el periodo 20002014. Coordinacion General de Producción y Productividad. Gerencia de Desarrollo de Plantaciones Forestales Comerciales. Secretaría de Medio Ambiente y Recursos Naturales. Recuperado de http://www.conafor.gob.mx:8080/documentos/docs/43/6019 Principales $\% 20$ especies $\% 20$ maderables $\% 20$ establecidas $\% 20$ en $\%$ 20PFC $\% 20$ por $\% 20$ Entidad $\% 20$ Federativa $\% 20$ en $\% 202000 \% 20$ $\% 202014$.pdf

Espinoza, L., Slaton, N., \& Mozaffari, M. (2012). Como interpretar los resultados de los análisis de suelos. Agricultura y Recursos Naturales. Cooperative Extension Service, Universidad de Arkansas, US Department of Agriculture, and county governments cooperating. Recuperado de https://www.uaex.edu/publications/PDF/FSA-2118SP.pdf

Fernández, L. C. L., \& Rojas, G. A. (2006). Análisis físicos y químicos en suelo. En L. C. Fernández., N. G. Rojas., T. G. Roldán., M. E. Ramírez., H. G. Zegarra., R. U. Hernández., R. J. Reyes., D. Flores \& J. M. Arce (Eds.), Manual de técnicas de análisis de suelos aplicadas a la remediación de sitios contaminados (pp. 19-80). México D. F.: Instituto Mexicano del Petroleo, Secretaría del Medio Ambiente y Recursos Naturales, Instituto Nacional de Ecología. Recuperado de http://biblioteca.semarnat.gob.mx/janium/Documentos/Ciga/ Libros2011/CG008215.pdf

García, E. (2004). Modificaciones al sistema de clasificación climática de Köppen. Universidad Nacional Autónoma de México. Recuperado de http://www.librosoa.unam.mx/handle/123456789/1372

Garrido, M. S. V. (1971). Interpretación de análisis de suelos. Investigaciones Geográficas. Madrid. España: Ministerio de Agricultura Pesca y Alimentación. Recuperado de https://www.mapa.gob.es/ministerio/pags/biblioteca/hojas/hd _1993_05.pdf

Gómez, M. (2005). Guía técnica para el manejo nutricional de los cultivos: Diagnóstico, interpretación y recomendación de planes de fertilización. Recuperado de https://www.google.com/search?rlz=1C1EJFA_enMX816MX8 16\&ei=LLAGXaeNBKeR0gLEw7WgBg\&q $=\mathrm{Gu} \% 25 \mathrm{C} 3 \% 25 \mathrm{~A}$ $\mathrm{Da}+\mathrm{T} \% 25 \mathrm{C} 3 \% 25 \mathrm{~A} 9$ cnica + para $+\mathrm{el}+$ manejo + nutricional $+\mathrm{de}+\mathrm{l}$ os + cultivos $\% 253 \mathrm{~A}+$ Diagn $\% 25 \mathrm{C} 3 \% 25 \mathrm{~B} 3$ stico $\% 252 \mathrm{C}+$ interpret aci $\% 25 \mathrm{C} 3 \% 25 \mathrm{~B} 3 \mathrm{n}+\mathrm{y}+$ recomendaci $\% 25 \mathrm{C} 3 \% 25 \mathrm{~B} 3 \mathrm{n}+\mathrm{de}+$ planes $+\mathrm{de}+$ ferti

Gonzáles, G. T., \& Serrano, J. R. M. (2004). Propiedades y utilizaciones de la madera de melina (Gmelina arborea Roxb) procedente de árboles plantados en Costa Rica. Revista Forestal Mesoamericana 
Kurú, 6(17), 61-69. Recuperado de http://revistas.tec.ac.cr/index.php/kuru/article/view/386

González-Rojas, M., Murillo-Cruz, R., \& Ávila-Arias, C. (2016). Crecimiento inicial de Gmelina arborea Roxb. aplicando diferentes tratamientos de fertilización en el Pacífico Sur de Costa Rica. Revista Forestal Mesoamericana Kurú, 13(33), 29. doi: 10.18845/rfmk.v13i33.2575

Guisande, C., Vaamonde, A., \& Barreiro, A. (2013). Tratamiento de datos con R, Statistica y SPSS. $2^{a}$ Edición electrónica. Ediciones Díaz de Santos, 1-977. Madrid, España. Recuperado de https://www.editdiazdesantos.com/libros/guisandetratamiento-de-datos-con-r-statistica-y-spss-L03009981001.html

Indira, E. P. (2006). Provenance variations in Gmelina arborea with particular reference to tree form. Journal of Tropical Forest Science, 36-50. Recuperado de https://www.jstor.org/stable/43594644

Instituto Nacional De Estadística Geografía e Informática [Inegi] (2009). Prontuario de información geográfica municipal de los Estados Unidos Mexicanos Tlatlaya, Estado de México Clave geoestadística 15105. México D. F.: Inegi.

Isebrands, J. G., \& Crow, T. R. (1975). Introduction to uses and interpretation of principal component analyses in forest biology. General Technical Report NC-17. St. Paul, MN: US Dept. of Agriculture, Forest Service, North Central Forest Experiment Station, 17. Recuperado de https://www.nrs.fs.fed.us/pubs/gtr/ gtr_nc017.pdf

Kumar, J. N., Patel, K., Kumar, R. N., \& Kumar, R. B. (2011). Forest structure, diversity and soil properties in a dry tropical forest in Rajasthan, Western India. Annals of Forest Research, 54(1), 89-98. Recuperado de https://www.afrjournal.org/index.php/afr/article/view/99

Mariño-Macana, Y. A., \& Rodríguez-Melo, M. A. (2010). El punto de marchitez permanente (PMP) en melina (Gmelina arborea L. Roxb) para la Costa Caribe colombiana ¿una característica para la selección de clones?. Revista Ciencia y Tecnología Agropecuaria, 11(2), 116-121. Recuperado de https://www.redalyc.org/ articulo.oa ?id=4499/449945029002

Martínez, H. A. (2015). Melina (Gmelina arborea Roxb.): condiciones para su cultivo "Fomento de la reforestación comercial para la mejora y conservación de las reservas de carbono.” Moravia, Costa Rica: Fondo Nacional de Financiamiento Forestal Forest Monitoring System for REDD+ Costa Rica. Recuperado de https://docplayer.es/74588165-Fondo-nacional-definanciamiento-forestal-forest-monitoring-system-for-reddcosta-rica.html

Martínez, J. A., Zuluaga, J. J. P., Romero, J. F., Baquero, C. M., Berrio, E. G., Arrieta, A. A., \& Pérez, D. H. (2008). Efecto de prácticas de manejo de suelos sobre el crecimiento de plantas de Gmelina arborea y Pachira quinata en Zambrano, Bolivar. En S. Caicedo, C. R. Salamanca, C. A. Jaramillo, E. F. Almansa, y D. M. Peña. (Eds.), XIV Congreso Colombiano de la Ciencia del Suelo (p. 108). Villavicencio, Meta. Colombia: Sociedad Colombiana de la Ciencia del Suelo.

Moya-Roque, R., Muñoz-Acosta, F., Salas-Garita, C., Berrocal-Jiménez, A., Leandro-Zúñiga, L., \& Esquivel-Segura, E. (2010). Tecnología de madera de plantaciones forestales: Fichas técnicas. Revista Forestal Mesoamericana Kurú, 7, 18-19. Recuperado de https://revistas.tec.ac.cr/index.php/kuru/article/view/383

Murillo, L. F., Hernández, X., \& Murillo, O. (1996). Evaluacion de la calidad de plantaciones de cipres (Cupressus lusitanica) en el valle de el Guarco, Costa Rica. Agronomia Costarricense, 20(1), 17-23.

Pritchett, W. L. (1986). Suelos forestales: propiedades, conservación y mejoramiento. México D. F.: Limusa. Recuperado de http://www.sidalc.net/cgi-

bin/wxis.exe/?IsisScript=PLAFOR.xis\&method=post\&formato $=2 \&$ cantidad $=1$ \&expresion $=\mathrm{mfn}=000077$

Protectora de Bosques del Estado de México [Probosque] (2019). Superficie plantada de Gmelina arborea (Roxb.) en el Estado de México. Metepec, Estado de México.: Probosque. Recuperado de http://probosque.edomex.gob.mx/plantaciones_forestales

Rejón, L. A. S., \& Romero, J. L. (2004). Plantations of Gmelina arborea in southern Mexico. New Forests, 28(2-3), 293-297. Recuperado de https://link.springer.com/article/10.1023/B:NEFO.000004095 6.68838 .97

Rodríguez, A., L. G. \& Castañeda, G. A. M. (2014). Carbon capture in three forest species in Meta Department. Revista Cientifica Guarracuco, 18(29), $10 . \quad$ Recuperado de http://revistas.unimeta.edu.co/index.php/rc_es_guarracuco/art icle/view/67/277

Rojas, F. R., \& Murillo, O. G. (2004). Botánica y ecología. En F. Rojas, D. Arias, R. Moya, A. Meza, O. Murillo, \& M. Arguedas (Eds.), Manual para productores de melina (Gmelina arborea) en Costa Rica (p. 314). Cártago, Costa Rica: Fonafifo. Recuperado de http://www.sirefor.go.cr/Documentos/Especies_plantaciones/ MELINA/Manual para los productores de melina.pdf\%5Cnhttp://www.sirefor.go.cr

Rubio, M. J. H., \& Berlanga, V. S. (2012). Cómo aplicar las pruebas paramétricas bivariadas $t$ de Student y ANOVA en SPSS. Caso práctico. Revista d'Innovació $i$ Recerca En Educació, 5(2), 83-100. doi: 10.1344/reire2012.5.2527

Ruiz-Blandon, B. A., Hernández-Álvarez, E., Rodríguez-Macias, R., \& Salcedo-Pérez, E. (2020). Valoración dasométrica y producción de biomasa en Gmelina arborea Roxb. ex Sm. establecida en 
plantaciones puras y mixtas. Revista mexicana de ciencias forestales, 11(59), 94-117.

Salcedo-Pérez, E., Blandon-Ruiz, B. A., Hernández-Álvarez, E., González-Cruz, R., Bernabé-Antonio, A., Orozco-Guareño, E., ... Delgado-Fornué, E. (2019). Propiedades del suelo y nitrógeno como indicadores del crecimiento en plantaciones comerciales de teca. Revista Mexicana de Ciencias Forestales, 10(52). doi: 10.29298/rmcf.v10i52.398

Salcedo-Pérez, E., Ypushima-Pinedo, A. L., González-Cruz, R., ZamoraNátera, J. F., Rodríguez-Macías, R., \& Sánchez-Hernández, R. (2014). Efecto de las propiedades edáficas y el contenido nutrimental foliar sobre el crecimiento de teca. Revista Mexicana de Ciencias Forestales, 5(24), 80-91. doi: 10.29298/rmcf.v5i24.321

Santana, J. S., \& Farfán, E. M. (2014). El arte de programar en R: un lenguaje para la estadística. Jiutepec, Morelos, México: Instituto Mexicano de Tecnología del Agua, UNESCO, Comité Nacional Mexicano del Programa Hidrológico Internacional. 197 p. Recuperado de https://cran.rproject.org/doc/contrib/Santana_El_arte_de_programar_en_R. pdf

Secretaría del Medio Ambiente y Recursos Naturales [Semarnat]. (2002). Norma Oficial Mexicana, Especificaciones de Fertilidad, Salinidad y Clasificación de Suelos, Estudio, Muestreo y Análisis. Recuperado de https://www.google.com/search?rlz=1C1EJFA_enMX816MX8 16\&ei=laUGXf6LEqaA0wKcrqf4Bg\&q=Norma + Oficial + Mexi cana $\% 2 \mathrm{C}+$ Especificaciones + de + Fertilidad $\% 2 \mathrm{C}+$ Salinidad $+\mathrm{y}+$ Clasificación+de+Suelos $\% 2 \mathrm{C}+$ Estudio $\% 2 \mathrm{C}+$ Muestreo+y+Aná lisis\&oq $=$ Norma + Oficial + Mexicana $\% 2 C+$ Esp

Thiers, O., \& Gerding, V. (2007). Variabilidad topográfica y edáfica en bosques de Nothofagus betuloides (Mirb) Blume, en el suroeste de Tierra del Fuego, Chile. Revista Chilena de Historia Natural, 80(2), 201-211. doi:10.4067/S0716-078X2007000200006

Vallejos, O. S. B. (1996). Productividad y relaciones del índice de sitio con variables fisiográficas edafoclimáticas y foliares para Tectona grandis LF, Bombacopsis quinatum (Jacq.) Dugand y Gmelina arborea Roxb, en Costa Rica. Centro Agronómico Tropical de Investigación y Enseñanza. Recuperado de http://www.sidalc.net/cgi-

$\mathrm{bin} /$ wxis.exe/?IsisScript $=$ orton. xis\&method $=$ post $\&$ formato $=2$ \&cantidad $=1$ \&expresion $=\mathrm{mfn}=062684$

Vásquez, W., \& Ugalde, L. (1996). Rendimiento y Calidad de Sitio para Gmelina arborea, Bombacopsis quinatum y Pinus caribaea en Guanacaste, Costa Rica. En X Congreso Nacional Agronómico / II Congreso de Suelos. (pp. 7-13). Costa Rica: Centro Agronómico Tropical de Investigación y enseñanza (CATIE). Recuperado de http://repositorio.bibliotecaorton.catie.ac.cr/bitstream/handle/ 11554/3819/Reendimiento_y_calidad_de_sitio_informe_final.p df? sequence $=1 \&$ isAllowed $=\mathrm{y}$

Wee, A. K. S., Li, C., Dvorak, W. S., \& Hong, Y. (2012). Genetic diversity in natural populations of Gmelina arborea: implications for breeding and conservation. New Forests, 43(4), 411-428. doi: $10.1007 / \mathrm{s} 11056-011-9288-2$

Manuscrito recibido el 19 de junio de 2019

Aceptado el 20 de marzo de 2020

Publicado el 19 de marzo de 2021

Este documento se debe citar como:

Telles A., R., Alanís R., E., Jiménez P., J., Aguirre C., O. A, \& y Garcia G., D. A. (2021). Características edáficas y topográficas asociadas con el crecimiento en volumen de Gmelina arborea Roxb, en Tlatlaya Estado de México. Madera y Bosques, 27(1), e2711987. doi: 10.21829/myb.2021.2711987

Madera y Bosques por Instituto de Ecología, A.C. se distribuye bajo una Licencia Creative Commons Atribución-NoComercialCompartirlgual 4.0 Internacional. 